\title{
Splitting potential and Poincaré-Melnikov method for whiskered tori in Hamiltonian systems
}

\author{
Amadeu Delshams and Pere Gutiérrez \\ Departament de Matemàtica Aplicada I \\ Universitat Politècnica de Catalunya \\ Diagonal 647, 08028 Barcelona \\ amadeu@ma1.upc.es, pereg@ma1.upc.es
}

3rd revised version - November 26, 1999

\begin{abstract}
Summary
We deal with a perturbation of a hyperbolic integrable Hamiltonian system with $n+1$ degrees of freedom. The integrable system is assumed to have $n$-dimensional hyperbolic invariant tori with coincident whiskers (separatrices).

Following Eliasson, we use a geometric approach closely related to the Lagrangian properties of the whiskers, to show that the splitting distance between the perturbed stable and unstable whiskers is the gradient of a periodic scalar function of $n$ phases, which we call splitting potential. This geometric approach works for both the singular (or weakly hyperbolic) case and the regular (or strongly hyperbolic) case, and provides the existence of at least $n+1$ homoclinic intersections between the perturbed whiskers.

In the regular case, we also obtain a first order approximation for the splitting potential, that we call Melnikov potential. Its gradient, the (vector) Melnikov function, provides a first order approximation for the splitting distance. Then the nondegenerate critical points of the Melnikov potential give rise to transverse homoclinic intersections between the whiskers. Generically, when the Melnikov potential is a Morse function, there exist at least $2^{n}$ critical points.

The first order approximation relies on the $n$-dimensional Poincaré-Melnikov method, to which an important part of the paper is devoted. We develop the method in a general setting, giving the Melnikov potential and the Melnikov function in terms of absolutely convergent integrals, which take into account the phase drift along the separatrix and the first order deformation of the perturbed hyperbolic tori. We provide formulas useful in several cases, and carry out explicit computations that show that the Melnikov potential is a Morse function, in different kinds of examples.
\end{abstract}

MSC numbers. 58F05, 34C37, 58F36, 34C 30, 70F15

PAC numbers. 45.20.Jj, 02.40.Vh, 05.45.-a, 95.10.Fh, 45.50.Pk

\section{Setup and introduction}

\subsection{Perturbation of a hyperbolic integrable Hamiltonian}

It is well-known that the problem of giving conditions for the splitting of the whiskers of hyperbolic invariant tori is one of the main difficulties related with the Arnold diffusion, a phenomenon of instability in perturbations of integrable Hamiltonian systems with more than 2 degrees of freedom. The present paper is concerned with the study of the existence of homoclinic orbits and splitting in a wide class of Hamiltonians. The tools used are a geometric approach based on Eliasson's work [Eli94], and the Poincaré-Melnikov method. 
We start with a perturbation of a hyperbolic integrable Hamiltonian, with $n+1 \geq 3$ degrees of freedom. In canonical variables $z=(x, y, \varphi, I) \in D \subset \mathbb{T} \times \mathbb{R} \times \mathbb{T}^{n} \times \mathbb{R}^{n}$, with the symplectic form $\mathrm{d} x \wedge \mathrm{d} y+\mathrm{d} \varphi \wedge \mathrm{d} I$, consider a real analytic Hamiltonian of the form

$$
\begin{aligned}
& H(x, y, \varphi, I ; \mu)=H_{0}(x, y, I)+\mu H_{1}(x, y, \varphi, I), \\
& H_{0}(x, y, I)=\langle\omega, I\rangle+\frac{1}{2}\langle\Lambda I, I\rangle+\frac{y^{2}}{2}+V(x)+\langle\lambda, I\rangle y,
\end{aligned}
$$

where $\mu$ is a perturbation parameter. The Hamiltonian equations associated to $H$ are:

$$
\begin{aligned}
& \dot{x}=y+\langle\lambda, I\rangle \quad+\mu \partial_{y} H_{1}(x, y, \varphi, I), \\
& \dot{y}=-V^{\prime}(x) \quad-\mu \partial_{x} H_{1}(x, y, \varphi, I), \\
& \dot{\varphi}=\omega+\Lambda I+\lambda y+\mu \partial_{I} H_{1}(x, y, \varphi, I), \\
& \dot{I}=\quad-\mu \partial_{\varphi} H_{1}(x, y, \varphi, I) .
\end{aligned}
$$

The given ingredients of $H_{0}$ are the vectors $\omega, \lambda \in \mathbb{R}^{n}$, the symmetric $(n \times n)$-matrix $\Lambda$, and the function $V(x)$ of $x \in \mathbb{T}$, which is required to have a unique and nondegenerate global maximum. To fix ideas, we require

$$
\begin{aligned}
& V(0)=0, \quad V^{\prime}(0)=0, \quad V^{\prime \prime}(0)<0 \\
& V(x)<0 \quad \forall x \neq 0 \quad(\bmod 2 \pi) .
\end{aligned}
$$

We also assume the following nondegeneracy condition:

$$
\operatorname{det}\left(\begin{array}{cc}
1 & \lambda^{\top} \\
\lambda & \Lambda
\end{array}\right) \neq 0
$$

The integrable Hamiltonian $H_{0}$ can easily be studied. Introducing

$$
P(x, y)=\frac{y^{2}}{2}+V(x), \quad \widehat{P}(x, y, I)=P(x, y+\langle\lambda, I\rangle),
$$

and

$$
\widehat{\Lambda}=\Lambda-\lambda \lambda^{\top}
$$

the unperturbed Hamiltonian $H_{0}$ can be rewritten as

$$
H_{0}=\langle\omega, I\rangle+\frac{1}{2}\langle\widehat{\Lambda} I, I\rangle+\widehat{P}(x, y, I)
$$

Note that the nondegeneracy condition (5) is equivalent to impose that $\operatorname{det} \widehat{\Lambda} \neq 0$. We see that, on every plane $I=$ const, the Hamiltonian $H_{0}$ reduces to a 1-degree-of-freedom Hamiltonian: a generalized pendulum (the standard pendulum being given by $V(x)=\cos x-1$ ). This pendulum has $(x, y)=$ $(0,-\langle\lambda, I\rangle)$ as a hyperbolic equilibrium point, with (homoclinic) separatrices given by $y+\langle\lambda, I\rangle=$ $\pm \sqrt{-2 V(x)}$. The Lyapunov exponents of the hyperbolic point are $\pm \alpha$, with

$$
\alpha=\sqrt{-V^{\prime \prime}(0)} .
$$

Therefore, the Hamiltonian $H_{0}$ has an $n$-parameter family of $n$-dimensional whiskered tori (or hyperbolic invariant tori) given by the equations $I=$ const, $y=-\langle\lambda, I\rangle, x=0$. The associated stable and unstable whiskers (or invariant manifolds) of each torus coincide, and hence all orbits on this (unique) whisker are homoclinic, i.e. biasymptotic to the torus. Our aim is to study the splitting of the whiskers for $\mu \neq 0$, detecting homoclinic intersections and measuring the splitting distance.

We will focus our attention on a concrete hyperbolic torus, that we assume located at the origin: $I=0, x=y=0$, with flow $\dot{\varphi}=\omega$. The vector of frequencies $\omega$ is assumed to satisfy a Diophantine condition: for some $\tau \geq n-1$ and $\gamma>0$,

$$
|\langle k, \omega\rangle| \geq \gamma|k|^{-\tau} \quad \forall k \in \mathbb{Z}^{n} \backslash\{0\},
$$


where $|k|=\sum_{j=1}^{n}\left|k_{j}\right|$. We recall that, for a fixed $\tau>n-1$, the set of vectors $\omega$ satisfying this condition for some $\gamma>0$ has full measure in $\mathbb{R}^{n}$.

In fact, it is convenient to allow $\omega$ to depend on an additional parameter $\varepsilon$, considering fast frequencies $\omega=\omega^{*} / \sqrt{\varepsilon}$. The parameters $\varepsilon$ and $\mu$ can be whether independent or linked by a relation of the type $\mu=\varepsilon^{p}$; these two cases will be called, respectively, regular and singular. The singular case is very important in the study of the stability in a general nearly-integrable Hamiltonian, in which the integrable system has no hyperbolicity, but the perturbation provides some weak hyperbolicity (see more details in section 1.3).

As a final remark, note that taking $\lambda=0$ in (2) we have an uncoupled unperturbed Hamiltonian $H_{0}$, which is somewhat simpler since it is formed by a pendulum and $n$ rotors. However, our approach is also addressed to the more general coupled case $\lambda \neq 0$. The motivation is that the coupling term $\langle\lambda, I\rangle y$ appears in a natural way when one considers the important case of a nearly-integrable Hamiltonian, in a region close to a single resonance (see section 1.3). It is shown at the end of section 3.4 that, making a symplectic change of variables, one is able to eliminate this coupling term, but then one gets a Hamiltonian that is non-periodic in $x$ (leading in this way to a heteroclinic problem).

\subsection{Parameterization of the unperturbed torus and its whiskers}

Before describing the results, we introduce some notations for the unperturbed Hamiltonian $H_{0}$. We denote $\mathcal{T}_{0}$ its $n$-dimensional hyperbolic invariant torus of frequency vector $\omega$. This torus is given by the equations $I=0, x=y=0$, and can obviously be parameterized by

$$
\mathcal{T}_{0}: \quad z_{0}^{*}(\varphi)=(0,0, \varphi, 0), \quad \varphi \in \mathbb{T}^{n} .
$$

Note that the trajectories on $\mathcal{T}_{0}$ are $t \mapsto z_{0}^{*}(\varphi+\omega t)$ for any given $\varphi$.

As mentioned in section 1.1, the stable and unstable whiskers of the torus $\mathcal{T}_{0}$ coincide; this homoclinic whisker is then called separatrix and is given by the equations $I=0, P(x, y)=0$. We denote $\mathcal{W}_{0}$ the positive part $(y>0)$ of the separatrix. To give a suitable parameterization for $\mathcal{W}_{0}$, we consider the 1-degree-of-freedom Hamiltonian $P(x, y)$, and denote $\left(x_{0}(s), y_{0}(s)\right)$ the associated homoclinic trajectory, biasymptotic for $s \rightarrow \pm \infty$ to the hyperbolic point $(0,0)$, with $x_{0}(0)=\pi, y_{0}(0)>0$. In fact, $x_{0}(s)$ goes from 0 to $2 \pi$ when $s$ goes from $-\infty$ to $\infty$. It is clear that we can then give the whisker $\mathcal{W}_{0}$ the parameterization

$$
\mathcal{W}_{0}: \quad z_{0}(s, \varphi)=\left(x_{0}(s), y_{0}(s), \varphi+\xi_{0}(s), 0\right), \quad s \in \mathbb{R}, \quad \varphi \in \mathbb{T}^{n}
$$

where the term

$$
\xi_{0}(s)=\left(x_{0}(s)-\pi\right) \lambda
$$

is included in view of the phase drift undergone by any trajectory when traveling along $\mathcal{W}_{0}$. This drift is associated to the coupling term. Note that, with our definition, $t \mapsto z_{0}(s+t, \varphi+\omega t)$ is a trajectory on $\mathcal{W}_{0}$ for any given $s, \varphi$. In other words, the dynamics on $\mathcal{W}_{0}$ is given by the equations $\dot{s}=1, \dot{\varphi}=\omega$.

It is clear that $z_{0}(s, \varphi) \in \mathcal{W}_{0}$ tends to $\mathcal{T}_{0}$ for $s \rightarrow \pm \infty$ : since $\lim _{s \rightarrow \pm \infty} \xi_{0}(s)= \pm \pi \lambda$, we have

$$
\lim _{s \rightarrow \pm \infty}\left[z_{0}(s, \varphi)-z_{0}^{*}(\varphi \pm \pi \lambda)\right]=0 .
$$

In fact, we can provide exponentially decreasing bounds in terms of the Lyapunov exponents $\pm \alpha$. For $\pm s$ big enough,

$$
\left|z_{0}(s, \varphi)-z_{0}^{*}(\varphi \pm \pi \lambda)\right| \leq C e^{\mp \alpha s}
$$

(we use the Euclidean norm for vectors). We deduce that every trajectory on $\mathcal{W}_{0}$ is biasymptotic to two different trajectories on the invariant torus $\mathcal{T}_{0}$ :

$$
\lim _{t \rightarrow \pm \infty}\left[z_{0}(s+t, \varphi+\omega t)-z_{0}^{*}(\varphi \pm \pi \lambda+\omega t)\right]=0,
$$

with exponentially decreasing bounds. Note that if $\lambda$ is an integer then the two trajectories on $\mathcal{T}_{0}$ coincide. 


\subsection{Regular and singular hyperbolic Hamiltonians}

When the frequency vector $\omega$ is fixed, the Hamiltonian introduced in (1-2) has the property that the hyperbolicity and the homoclinic orbits are present in the unperturbed Hamiltonian $(\mu=0)$. Thus, we have a regular (or nonsingular) system, often referred to as strongly hyperbolic.

Eventually, we can allow $\omega$ to depend on an additional parameter: $\omega=\omega(\varepsilon)$. For instance, we can consider fast frequencies $\omega=\omega^{*} / \sqrt{\varepsilon}$, where $\varepsilon>0$ is a small parameter (and then $\gamma=\gamma^{*} / \sqrt{\varepsilon}$ in (10)). Then if we let $\varepsilon \rightarrow 0^{+}$and consider $\mu=\varepsilon^{p}$ for some $p>0$, the system becomes singular or weakly hyperbolic. This case can also be called totally singular, because all the frequencies are fast (in contrast with other problems that arise in celestial mechanics, where the frequencies have different time scales, like for instance $\omega=\left(\omega_{1}^{*}, \omega_{2}^{*} / \sqrt{\varepsilon}\right)$; see [CG94, RW98, GGM99]). We recall that, according to the terminology introduced in [CG94], the regular and singular systems are also said to be a-priori unstable and a-priori stable, respectively.

As a remark, assuming $H_{1}=H_{1}(x, \varphi)$ and performing the (noncanonical) linear change obtained by replacing $y, I$ by $y / \sqrt{\varepsilon}, I / \sqrt{\varepsilon}$, plus a change of time scale by a factor $\sqrt{\varepsilon}$, the Hamiltonian equations (3) are transformed in new equations associated to the Hamiltonian

$$
\left\langle\omega^{*}, I\right\rangle+\frac{1}{2}\langle\Lambda I, I\rangle+\frac{y^{2}}{2}+\varepsilon V(x)+\langle\lambda, I\rangle y+\varepsilon \mu H_{1}(x, \varphi),
$$

in which the hyperbolicity disappears for $\varepsilon \rightarrow 0^{+}$. This is a generalization of Lochak's example [Loc92, $\S \mathrm{V} 2]$, which corresponds to the case $V(x)=\cos x-1, \Lambda=\mathrm{Id}, \lambda=0$, and $H_{1}(x, \varphi)=(\cos x-1) f(\varphi)$ with some even function $f(\varphi)$.

In fact, Lochak's example is, in its turn, a generalization of the famous Arnold's example [Arn64] to an autonomous Hamiltonian of an arbitrary number of degrees of freedom, but with the important feature that the perturbation includes harmonics in $\varphi$ of arbitrarily high orders. It is worth recalling that the Arnold's example was the first illustration for the transition chains mechanism in order to establish the existence of diffusion in Hamiltonian systems with more than 2 degrees of freedom.

Note that, even in the case of fast frequencies, if one regards $\varepsilon$ and $\mu$ as independent parameters, keeping $\varepsilon>0$ fixed and letting $\mu \rightarrow 0$, then one still has a regular system. This strategy was introduced in [Arn64] in order to avoid dealing with a singular perturbation problem. In this case, the PoincaréMelnikov method can be applied directly to the detection of the splitting, provided the parameter $\mu$ is taken exponentially small with respect to $\varepsilon$ (this is due to that the Melnikov integrals are exponentially small in $\varepsilon$ ).

The singular case arises properly when the parameters $\varepsilon$ and $\mu$ satisfy a power-like relation of the type $\mu=\varepsilon^{p}$ (the smaller $p$ the better), and one lets $\varepsilon \rightarrow 0^{+}$. In this case, the problem of detecting the splitting from the Melnikov integrals is much more intricate, because of the exponentially small character of the integrals involved. However, it is believed that, under some weak conditions, the Melnikov integrals give the right predictions for the splitting.

It is important to stress that the study of a singular system is closely related to the general problem of stability in a nearly-integrable Hamiltonian system $H(\varphi, I)=h(I)+\varepsilon f(\varphi, I)$, in angle-action variables $(\varphi, I) \in \mathbb{T}^{n+1} \times \mathbb{R}^{n+1}$ (here, the perturbation parameter is $\varepsilon$ ). Assume, for a given action $I^{*}$, that the associated frequency vector has a single resonance: for instance, $\partial_{I} h\left(I^{*}\right)=\left(0, \omega^{*}\right)$ with $\omega^{*} \in \mathbb{R}^{n}$ nonresonant. As shown in [DG98] (see also [Eli94, RW97]), one step of resonant normal form procedure can be performed near $I^{*}$ and leads, under some generic hypotheses and after a scaling, to a hyperbolic Hamiltonian of the type (1-4), taking as $H_{0}$ the truncated normal form. One has:

$$
\omega=\frac{\omega^{*}}{\sqrt{\varepsilon}}, \quad \mu=\mathrm{O}\left(\varepsilon^{1 / 2}\right)
$$

and this relation between $\varepsilon$ and $\mu$ says that this system is singular (to have a regular system, one should consider $\varepsilon>0$ fixed and allow $\mu \rightarrow 0$ ). We point out also that, in general, the truncated normal form $H_{0}$ includes a coupling term: $\lambda \neq 0$ in (2).

In fact, the detection of the (homoclinic) splitting between the whiskers of hyperbolic tori in the singular case is an important step but it is not the only difficulty related with the study of Arnold diffusion through transition chains. Indeed, other related difficulties are the study of the transition 
properties of the tori, the detection of heteroclinic intersections between whiskers of different tori, and jumping the gaps associated to double resonances.

\subsection{Description of the results}

The main goal of the present paper is to introduce a geometric method to measure the splitting of the whiskers in our Hamiltonian (1-2), relating it with the Melnikov function.

To such end, we first have to establish the surviving of the whiskered torus of frequency vector $\omega$, as well as its local whiskers, and then we can extend them to global whiskers in order to compare the stable and the unstable ones. The surviving of the torus and its local whiskers under a small perturbation can be ensured by means of a hyperbolic KAM theorem, a version of the KAM theorem adapted to this problem. We point out that the use of KAM theory cannot be avoided in this general setting, in which we consider an arbitrary perturbation $H_{1}$. Under more restrictive hypotheses on $H_{1}$, KAM theory may not be necessary (see for instance [Sau99]).

The hyperbolic KAM theorem is dealt in most papers considering local variables around the whiskered torus. A different approach was introduced by Eliasson [Eli94], by rewriting the theorem in the original variables, global in $x \in \mathbb{T}$. This allowed him to introduce in a very natural way a (vector) function measuring the splitting distance between the perturbed whiskers. Another key fact, not discussed before [Eli94], is the exploitment of exact symplectic transformations to normal form in the hyperbolic KAM theorem; this is crucial in order to establish the existence of homoclinic intersections between the whiskers, in both regular and singular systems (although the splitting was not computed). Similar results can be inferred from the work [Bol95].

Our main result is that, taking suitable variables, the splitting distance can be put as the gradient of some scalar periodic function, that we call splitting potential. This function leads to two consequences. First, the result of [Eli94] on the existence of homoclinic orbits is recovered; this holds for regular and singular systems. Second, using the Poincaré-Melnikov method we can go farther than [Eli94]: we get the Melnikov potential as a first order approximation for the splitting potential, though in this paper this result is good enough only for the regular case. However, it is expected that this approximation also holds in the singular case $\mu=\varepsilon^{p}$ for some suitable $p>0$. In this sense, our results can be considered as a first step in the study of the singular case.

Let us turn now to describe the contents of the present paper. In section 2.1, we give a statement (theorem 1) of Eliasson's version [Eli94] of the hyperbolic KAM theorem, providing a symplectic transformation $\Phi$ into a local normal form $\widetilde{H}=H \circ \Phi$, having a simpler expression in which the perturbed torus becomes transparent, as well as its whiskers. We are interested in a normal form defined in a whole neighborhood of the torus, as in [Eli94, Nie99], according to the Kolmogorov's approach to KAM theory.

In section 2.2, the expression of $\widetilde{H}$ provided by KAM theory allows us to take parameters $s, \varphi$ on the perturbed local whiskers, analogous to the parameters of the unperturbed whiskers introduced in section 1.2. These parameterizations are extended to the global whiskers in section 2.3, and are useful in measuring the splitting distance.

We have designed section 3 in order to be useful to the non-expert reader. In section 3.1, we develop the Poincaré-Melnikov method for the multidimensional case, and give a first order approximation for the splitting of the whiskers of hyperbolic tori. Taking into account the Hamiltonian character of the equations, it turns out that the (vector) Melnikov function $M(\varphi)$ is simply the gradient of a scalar function $L(\varphi)$, which we call the Melnikov potential: $M(\varphi)=\partial_{\varphi} L(\varphi)$. We point out that $L$ and $M$ are given by absolutely convergent integrals (see formulas (31-32)), thanks to the fact that the phase drift along the separatrix (due to the nonzero coupling term) and the first order deformation of the perturbed hyperbolic tori are taken into account. In the uncoupled case $\lambda=0$, the formula for the Melnikov potential is somewhat simpler, and takes the form:

$$
L(\varphi)=-\int_{-\infty}^{\infty}\left[H_{1}\left(z_{0}(t, \varphi+\omega t)\right)-H_{1}\left(z_{0}^{*}(\varphi+\omega t)\right)\right] \mathrm{d} t+\text { const. }
$$

We see (proposition 5) that, in the regular case, the Melnikov function $M$ provides a first order approximation for the splitting distance (measured along the $I$-direction). Thus, in this regular case, the nondegenerate critical points of $L$ give rise to transverse homoclinic orbits. In particular, when $L$ is a 
Morse function (a generic property), there exist at least $2^{n}$ transverse homoclinic orbits for $|\mu|$ small enough.

To illustrate the properties of the Melnikov potential, some examples are considered in sections 3.2 and 3.3, showing the existence of exactly $2^{n}$ transverse homoclinic orbits. As a measure of the transversality of the homoclinic intersections, we also compute the determinant of the symmetric matrix $\partial_{\varphi} M=\partial_{\varphi}^{2} L$ at the critical points of $L(\varphi)$. The example considered in section 3.3 is singular (with fast frequencies); this forces us to study (as a function of $\varepsilon$ ) the dominant harmonic of the Melnikov potential.

On the other hand, the formulation of the Poincaré-Melnikov method in the heteroclinic case is discussed in section 3.4, stressing the differences with the homoclinic case, the one mainly considered in this paper.

The aim of the following sections is to show that, using suitable variables, the "whole" splitting distance (and not only its first order approximation) is the gradient of some function, in order to establish the existence of homoclinic orbits even in the singular case. In section 4, we introduce flow-box variables in some real neighborhood containing a piece (local in $s$ but global in $\varphi$ ) of the stable and unstable whiskers; in this way the properties of these whiskers appear much more transparently. In particular, we stress that their splitting distance becomes a quasiperiodic function, only depending on $\varphi-\omega s$.

In section 5.1, we take advantage of the special formulation of theorem 1 (that uses the original variables) and introduce in some neighborhood an exact symplectic map between the global perturbed whiskers, taking the stable whisker onto the unstable one. So we proceed as in [Eli94], but we go farther because we express this map in the flow-box variables. In this way, the unstable whisker can be seen as a graphic over the stable one.

In section 5.2, the exactness of the symplectic map that links the two whiskers allows us to introduce in (84) a scalar function $\mathcal{L}(S, \psi)$, called splitting potential, as well as its gradient $\mathcal{M}(S, \psi)=\partial_{\psi} \mathcal{L}(S, \psi)$, called splitting function. We establish (theorem 10) that the functions $\mathcal{L}$ and $\mathcal{M}$ only depend on $\psi-\omega S$ and that, after a suitable reparameterization of the whiskers, the function $\mathcal{M}$ gives a measure for the splitting (the new parameters $S, \psi$ substitute the initial ones $s, \varphi$ on the whiskers). It becomes clear that the result on the splitting potential is very related to the Lagrangian properties of the whiskers. As a consequence, we deduce the existence of at least $n+1$ homoclinic orbits (not necessarily transverse), and this holds also in the singular case.

Finally, we obtain (theorem 11) first order approximations, valid for the regular case, for both the functions $\mathcal{L}$ and $\mathcal{M}$, in terms of the Melnikov potential $L$ and the Melnikov function $M$ introduced in section 3.1:

$$
\mathcal{L}(S, \psi)=\mu L(\psi-\omega S)+\mathrm{O}\left(\mu^{2}\right), \quad \mathcal{M}(S, \psi)=\mu M(\psi-\omega S)+\mathrm{O}\left(\mu^{2}\right) .
$$

We finish this introduction with some words about the computational aspects of the singular case. Theorem 11 provides an $\mathrm{O}\left(\mu^{2}\right)$ error term that is not small enough in the singular case $\mu=\varepsilon^{p}$ with $p>0$, due to the fact that the functions $L$ and $M$ are exponentially small with respect to $\varepsilon$. (This is illustrated in the example of section 3.3.)

To get better bounds for the error term between the splitting and Melnikov functions for real values, one should get a bound similar to that of theorem 11, but for a complex strip of the variables. Indeed, it is possible to carry out a control on the loss $\delta$ of complex domain in the angle variables in the normal form theorem, providing a more precise version of theorem 1 . Such a control on $\delta$ for a Hamiltonian like (1-2) has been done in [Nie99]. It still lacks an extension theorem and the flow-box variables extended to a suitable complex domain, to obtain exponentially small asymptotics for the splitting of separatrices, provided by the Melnikov function. This topic is currently being researched by the authors, and as a matter of fact, this is the strategy followed in [DS97, DGJS97], in simpler situations in which the normal form is integrable and the flow-box variables can be defined explicitly.

It is expected that under some general hypotheses on the perturbation, the first order approximation provided by the Poincaré-Melnikov method will give the dominant part of the splitting of the separatrices in the singular case. Such a result was announced in [RW98], but it still lacks a complete proof.

However, during the revision of this paper, we became aware of several recent preprints that contain essential advances on the asymptotics of the exponentially small splitting of separatrices taking place in single resonances of nearly-integrable Hamiltonian systems. Among them, the preprints [Sau99, LMS99, 
RW99] are based on using the Hamilton-Jacobi equation to the whiskers to exploit in a very transparent way their exact Lagrangian properties, and the preprint [PT99] introduces a different technique to attack this problem, using the method of continuous averaging.

\section{The perturbed torus and its whiskers}

\subsection{The hyperbolic KAM theorem in the original variables}

We are concerned in this section with the surviving of our Diophantine whiskered torus under perturbations, as well as its local stable and unstable manifolds. We call this result the hyperbolic KAM theorem. It follows from a convergent KAM-like iterative scheme, providing a symplectic transformation taking our Hamiltonian into a local normal form in some domain, in which the perturbed torus and its whiskers become transparent.

The first versions of the hyperbolic KAM theorem were proved in [Gra74, Zeh76] for a regular hyperbolic Hamiltonian. Subsequent papers, like [Tre91, CG94, Eli94, RW97, Val98, Nie99] (among others), provide proofs that hold also for a singular Hamiltonian. Nevertheless, significantly different approaches to KAM theory are followed in these papers: the Kolmogorov's approach (ensuring the surviving of one concrete Diophantine torus), and the Arnold's approach (producing a large Cantor family of surviving tori). These two approaches were followed initially for the standard KAM theorem in [Kol78, BGGS84] and in [Arn63, Pös82], respectively, and have both been translated to the hyperbolic context.

Among the quoted papers, we follow here the approach of [Eli94, Nie99], which are close to the original Kolmogorov's approach, in the sense that the normalizing transformation converges on a whole neighborhood of the torus, making possible the study of the dynamics near the torus and the local whiskers. In particular, this will allow us to control a neighborhood of the local stable whisker, which can be ensured in this way to contain also a piece of the global stable whisker (see section 5).

We also point out that most of the papers quoted above give the hyperbolic KAM theorem in terms of some local variables $(u, v, \varphi, I)$, sometimes called hyperbolic variables, in which the whiskers become coordinate planes, in a neighborhood (of some radius $r_{0}>0$ ) around the whiskered torus. Denoting $\Gamma$ the change to the hyperbolic variables, our starting Hamiltonian (1-2) can be written $G=G_{0}+\mu G_{1}$, with

$$
\begin{aligned}
& G_{0}(u, v, I)=H_{0} \circ \Gamma=\langle\omega, I\rangle+\frac{1}{2}\langle\widehat{\Lambda} I, I\rangle+\alpha u v+\mathrm{O}_{2}(u v), \\
& G_{1}(u, v, \varphi, I)=H_{1} \circ \Gamma
\end{aligned}
$$

The change $\Gamma$ comes from the well-known Moser's theorem [Mos56] (see also [CG94, §A3]) on the convergence of the Birkhoff normal form for a 1-degree-of-freedom Hamiltonian near a hyperbolic equilibrium point. In this way, the pendulum $P$ can be taken to a function of $u v$, and recall that $\alpha>0$ is the Lyapunov exponent, defined in (9).

The hyperbolic KAM theorem expressed in the hyperbolic variables provides, under the suitable conditions, a symplectic transformation $\Upsilon$ to normal form:

$$
\widetilde{G}=G \circ \Upsilon=\mathrm{const}+\langle\omega, I\rangle+\tilde{\alpha} u v+\mathrm{O}_{2}(u v, I) .
$$

In a further step from this habitual framework that uses the hyperbolic variables, Eliasson rewrote the hyperbolic KAM theorem and expressed it directly in the "original variables" [Eli94, p. 65]. This is very suitable to our purpose of carrying out a global control of the whiskers in order to study their splitting (see section 5.1).

Besides, another important fact in Eliasson's paper [Eli94] is that he takes advantage of the properties of the exact symplectic transformations that appear in the construction of the normal form. This tool is very useful in order to detect homoclinic intersections, because it will allow us to put the splitting function as the gradient of a splitting potential (see section 5.2). To recall what an exact symplectic transformation is, consider the 1-form

$$
\eta=-(y \mathrm{~d} x+I \mathrm{~d} \varphi)
$$


whose differential is the standard symplectic 2-form:

$$
\mathrm{d} \eta=\mathrm{d} x \wedge \mathrm{d} y+\mathrm{d} \varphi \wedge \mathrm{d} I .
$$

A transformation $\Phi$ is symplectic if the 1-form $\Phi^{*} \eta-\eta$ is closed, and it is exact symplectic if this 1-form is exact $(=\mathrm{d} S$, globally, for some scalar primitive $S)$.

As a simple example, a translation $T_{a}:(u, v, \varphi, I) \mapsto(u, v, \varphi, I-a)$ is symplectic but not exact symplectic if $a \neq 0$. Indeed, its primitive would be $\langle a, \varphi\rangle$, but this function cannot be defined globally for $\varphi \in \mathbb{T}^{n}$, because it is not periodic in $\varphi$.

In [Eli94], a translation $T_{a}$ is carried out on the normal form (14), with a suitable $a \in \mathbb{R}^{n}$ such that $\Upsilon \circ T_{a}$ is exact symplectic. This transformation is translated from the hyperbolic variables to the original ones: $\Phi=\Gamma \circ \Upsilon \circ T_{a} \circ \Gamma^{-1}$. Then the Hamiltonian $\widetilde{H}=H \circ \Phi$ can be taken as the normal form for $H$. This provides the result of [Eli94, p. 65], which we state below as theorem 1.

On the other hand, the very recent paper by Niederman [Nie99] deals with a similar framework, and provides a more refined version of the hyperbolic KAM theorem (in the hyperbolic variables), concerning the loss $\delta$ of complex domain in the angle variables $\varphi$. Although this control on $\delta$ is not necessary in the present paper, it is important in obtaining asymptotic estimates for the splitting in the singular case (taking $\delta$ as some power of the perturbation parameter $\varepsilon$ ), as we plan to do in the next future. The paper [Nie99] also works with exact symplectic transformations and, as a difference, the nondegeneracy condition imposed is the isoenergetic one instead of (5).

In fact, the control on the loss $\delta$ was previously carried out in some papers that deal with somewhat different contexts: in [RW97] for the Arnold's approach to the hyperbolic KAM theorem, and in [DGJS97] for fast quasiperiodic perturbations of a pendulum. Later, this feature is applied in an extension theorem and then exponentially small asymptotic estimates for the splitting are obtained (see [DS97, DGJS97, RW98]).

In this work, we define for $r>0$ the complex domain

$$
B_{r}=\{(x, y, \varphi, I):|x|,|y|,|I|,|\operatorname{Im} \varphi| \leq r\} .
$$

For a function $f(x, y, \varphi, I)$ analytic on some domain $D$ (and continuous on its closure), we denote $|f|_{D}$ its supremum norm.

In the whole paper, we will denote $C, C_{1}, C_{2}, \ldots$ some suitable (big enough) positive constants not depending on $\omega, \mu$. These constants will be relabeled along the paper. We point out that this dependence on $\omega, \mu$ is considered apart because these amounts depend on $\varepsilon$ in the singular case. We will also write $f=\mathrm{O}(g)$ if we can bound $|f| \leq C|g|$.

Theorem 1 Let $H=H_{0}+\mu H_{1}$ as described in (1-4), analytic on $B_{r}\left(r \leq r_{0}\right)$. Assume the frequency vector $\omega$ satisfies the Diophantine condition (10) for some $\tau>n-1$ and $\gamma>0$. Assume also the nondegeneracy condition (5). Then for $|\mu| \leq 1 / C$, there exists an exact symplectic transformation $\Phi=\Phi(\cdot ; \mu): B_{\nu r} \longrightarrow B_{r}$ (analytic with respect to $(x, y, \varphi, I)$ and $\mu$ ), $0<\nu<1$, and there exist $a=a(\mu), b=b(\mu)$ (analytic in $\mu$ ), such that $\widetilde{H}=H \circ \Phi$ takes the form

$$
\widetilde{H}=\text { const }+\langle\omega, I-a\rangle+b \widehat{P}(x, y, I)+\mathrm{O}_{2}(\widehat{P}(x, y, I), I-a) .
$$

Besides, the following estimates hold:

$$
|\Phi-\mathrm{id}|_{B_{\nu r}}=\mathrm{O}(\mu), \quad|a|,|b-1|=\mathrm{O}(\mu) .
$$

The constants $r_{0}, \nu, C$ do not depend on $\omega, \mu$.

In order to keep a more readable notation, we shall not make explicit the $\mu$-dependence of $\Phi, a, b$. Remarks.

1. The normal form $\widetilde{H}$ is not integrable in general, because it is nothing but rewriting the original Hamiltonian $H$ in other variables (without trucating it). Nevertheless, it is clear from the structure of $\widetilde{H}$ that it has a whiskered torus and the associated local whiskers, as we detail in the next section. 
2. The price paid for obtaining an exact symplectic transformation in theorem 1 is that the torus, in the normal form variables, is shifted from $I=0$ to $I=a$.

3. The most important point about theorem 1 is that, thanks to the use of the variables $x, y$ instead of the hyperbolic variables, the local normal form $\widetilde{H}$ can be put in terms of the generalized pendulum $\widehat{P}(x, y, I)$. We use intensively this feature in section 5.1 .

4. The validity of this result in the singular case, with $\mu=\varepsilon^{p}$ and $\omega=\omega^{*} / \sqrt{\varepsilon}$, can be established from the fact that the associated constants do not depend on $\omega, \mu$. Then the theorem applies in the singular case provided $|\varepsilon|$ is small enough.

\subsection{Parameterization of the perturbed torus and its local whiskers}

The local normal form $\widetilde{H}$ given in (16) has a hyperbolic invariant torus of frequency vector $\omega$. This torus and its associated local whiskers $\widetilde{\mathcal{W}}_{\text {loc }}^{+}$(stable) and $\widetilde{\mathcal{W}}_{\text {loc }}^{-}$(unstable) can be parameterized as follows:

$$
\begin{array}{ll}
\tilde{\mathcal{T}}: & \tilde{z}^{*}(\varphi)=(0,-\langle\lambda, a\rangle, \varphi, a), \quad \varphi \in \mathbb{T}^{n}, \\
\widetilde{\mathcal{W}}_{\text {loc }}^{ \pm}: & \tilde{z}(s, \varphi)=\left(x_{0}(b s), y_{0}(b s)-\langle\lambda, a\rangle, \varphi+\xi_{0}(b s), a\right), \quad \pm s \geq s_{0}, \quad \varphi \in \mathbb{T}^{n}
\end{array}
$$

(recall the drift term defined in (11)), with a suitable $s_{0}=s_{0}(r)$. Indeed, in order to have $\tilde{z}(s, \varphi) \in B_{\nu r}$, we need $\left|x_{0}(b s)\right|,\left|y_{0}(b s)\right|,|a| \leq \nu r$. These inequalities hold for $|s| \geq s_{0}$ with some $s_{0}$ only depending on $r$. In the parameters $s, \varphi$, the dynamics of $\widetilde{H}$ on $\widetilde{\mathcal{W}}_{\text {loc }}^{ \pm}$is given by $\dot{s}=1, \dot{\varphi}=\omega$. We will assume that the meaningless additive constant in (16) has been chosen in such a way that $\widetilde{H}\left(\widetilde{\mathcal{W}}_{\text {loc }}^{ \pm}\right)=0$.

We then have, for the original perturbed Hamiltonian $H$, a hyperbolic torus and its associated local stable and unstable whiskers:

$$
\begin{array}{cl}
\mathcal{T}: & z^{*}(\varphi)=\Phi\left(\tilde{z}^{*}(\varphi)\right), \quad \varphi \in \mathbb{T}^{n}, \\
\mathcal{W}_{\text {loc }}^{ \pm}: & z_{\text {loc }}^{ \pm}(s, \varphi)=\Phi(\tilde{z}(s, \varphi)), \quad \pm s \geq s_{0}, \quad \varphi \in \mathbb{T}^{n} .
\end{array}
$$

In the following lemma, to be used in section 3.1, we give a first order approximation in $\mu$ for the shift of the perturbed torus $\mathcal{T}$ with respect to the unperturbed torus $\mathcal{T}_{0}$, along the $I$-component. Thus, we measure $I^{*}(\varphi)$ (the $I$-component of $z^{*}(\varphi)$ ). To describe this approximation, we consider the (zero average) scalar function $\chi(\varphi)$ solving the following small divisors equation:

$$
\left\langle\omega, \partial_{\varphi} \chi\right\rangle+\overline{H_{1}}(0,0,0)=H_{1}(0,0, \cdot, 0),
$$

where the notation $\bar{f}$ denotes the $\varphi$-average of a function $f$. It is a well-known fact that the Diophantine condition (10) ensures the existence of the solution $\chi$ (see for instance [Rüs75]; in fact this goes back to [Kol78]). The use of $\chi$ as a first approximation for the perturbed torus was introduced by Treschev [Tre94].

Lemma 2 For any $\varphi \in \mathbb{T}^{n}$, one has

$$
I^{*}(\varphi)=\mu\left(\zeta-\partial_{\varphi} \chi(\varphi)\right)+\mathrm{O}\left(\mu^{2}\right)
$$

where we define

$$
\zeta=-\widehat{\Lambda}^{-1}\left(\overline{\partial_{I} H_{1}-\lambda \partial_{y} H_{1}}\right)(0,0,0) .
$$

Proof. Let us write $z^{*}(\varphi)=z_{0}^{*}(\varphi)+\mu z_{1}^{*}(\varphi)+\mathrm{O}\left(\mu^{2}\right)$. It is clear that $I_{0}^{*}(\varphi)=0$, and we have to find $I_{1}^{*}(\varphi)$. The Hamiltonian $H$ has $z^{*}(\varphi+\omega t)$ as a quasiperiodic trajectory densely filling the invariant torus $\mathcal{T}$. Note that $\frac{\mathrm{d}}{\mathrm{d} t} z^{*}(\varphi+\omega t)=\left\langle\omega, \partial_{\varphi} z^{*}(\varphi+\omega t)\right\rangle$ (we are using the notation $\left\langle\omega, \partial_{\varphi} f\right\rangle=\sum_{j=1}^{n} \omega_{j} \partial_{\varphi_{j}} f$ also for a vector function $f(\varphi))$; therefore $z^{*}(\varphi)$ satisfies the following partial differential equation:

$$
\left\langle\omega, \partial_{\varphi} z^{*}(\varphi)\right\rangle=\mathcal{J} \nabla H\left(z^{*}(\varphi)\right),
$$


where $\mathcal{J}$ denotes the standard symplectic matrix. We can write this equation in components (disregarding the $y$-component, that is not needed here):

$$
\begin{aligned}
& \left\langle\omega, \partial_{\varphi} x^{*}(\varphi)\right\rangle=y^{*}(\varphi)+\left\langle\lambda, I^{*}(\varphi)\right\rangle+\mu \partial_{y} H_{1}\left(z^{*}(\varphi)\right), \\
& \left\langle\omega, \partial_{\varphi} \varphi^{*}(\varphi)\right\rangle=\omega+\Lambda I^{*}(\varphi)+\lambda y^{*}(\varphi)+\mu \partial_{I} H_{1}\left(z^{*}(\varphi)\right), \\
& \left\langle\omega, \partial_{\varphi} I^{*}(\varphi)\right\rangle=-\mu \partial_{\varphi} H_{1}\left(z^{*}(\varphi)\right)
\end{aligned}
$$

(in the last component, we have used implicitly that the $n$ components of $I$ are first integrals of $H_{0}$ ). Expanding in $\mu$ up to first order, we obtain:

$$
\begin{aligned}
& \left\langle\omega, \partial_{\varphi} x_{1}^{*}(\varphi)\right\rangle=y_{1}^{*}(\varphi)+\left\langle\lambda, I_{1}^{*}(\varphi)\right\rangle+\partial_{y} H_{1}\left(z_{0}^{*}(\varphi)\right), \\
& \left\langle\omega, \partial_{\varphi} \varphi_{1}^{*}(\varphi)\right\rangle=\Lambda I_{1}^{*}(\varphi)+\lambda y_{1}^{*}(\varphi)+\partial_{I} H_{1}\left(z_{0}^{*}(\varphi)\right), \\
& \left\langle\omega, \partial_{\varphi} I_{1}^{*}(\varphi)\right\rangle=-\partial_{\varphi} H_{1}\left(z_{0}^{*}(\varphi)\right) .
\end{aligned}
$$

Note that (27) is a (vector) small divisors equation for $I_{1}^{*}(\varphi)$. This equation is of the same type as (19), but now with a zero average function in the right hand side. In fact, taking a $\varphi$-derivative in (19) we get

$$
\left\langle\omega, \partial_{\varphi}^{2} \chi(\varphi)\right\rangle=\partial_{\varphi}\left(\left\langle\omega, \partial_{\varphi} \chi(\varphi)\right\rangle\right)=\partial_{\varphi} H_{1}\left(z_{0}^{*}(\varphi)\right) .
$$

Comparing this equation to (27), we obtain the equality $I_{1}^{*}(\varphi)-\overline{I_{1}^{*}}=-\partial_{\varphi} \chi(\varphi)$.

To determine $\zeta=\overline{I_{1}^{*}}$, we take average parts in $(25-26)$ :

$$
\begin{aligned}
& \overline{y_{1}^{*}}+\left\langle\lambda, \overline{I_{1}^{*}}\right\rangle+\overline{\partial_{y} H_{1}\left(z_{0}^{*}(\cdot)\right)}=0, \\
& \Lambda \overline{I_{1}^{*}}+\lambda \overline{y_{1}^{*}}+\overline{\partial_{I} H_{1}\left(z_{0}^{*}(\cdot)\right)}=0 .
\end{aligned}
$$

Solving these linear equations, and recalling the matrix $\widehat{\Lambda}$ introduced in (7), we obtain (21).

We have to check also that the constant involved in the $\mathrm{O}\left(\mu^{2}\right)$-term in $(20)$ can be understood in the sense described just before theorem 1 . We see from (24) and (27) that the difference $I^{*}(\varphi)-\mu I_{1}^{*}(\varphi)$ satisfies the small divisors equation

$$
\left\langle\omega, \partial_{\varphi}\left(I^{*}(\varphi)-\mu I_{1}^{*}(\varphi)\right)\right\rangle=-\mu\left[\partial_{\varphi} H_{1}\left(z^{*}(\varphi)\right)-\partial_{\varphi} H_{1}\left(z_{0}^{*}(\varphi)\right)\right] .
$$

The right hand side of this equality is clearly $\mathrm{O}\left(\mu^{2}\right)$ for $\varphi \in \mathbb{T}^{n}$, according to (17) and the definition of $z^{*}(\varphi)$. Then the solution of $(28)$, without its average part, satisfies the bound

$$
\left(I^{*}(\varphi)-\mu I_{1}^{*}(\varphi)\right)-\left(\overline{I^{*}}-\mu \overline{I_{1}^{*}}\right)=\mathrm{O}\left(\mu^{2}\right)
$$

(with $\gamma$ as a denominator, but this is a constant). Using (22-23) and (25-26), we can also obtain a bound for the average part: $\overline{I^{*}}-\mu \overline{I_{1}^{*}}=\mathrm{O}\left(\mu^{2}\right)$, and we then get the right bound for the $\mathrm{O}\left(\mu^{2}\right)$-term in (20).

Remarks.

1. We could also have deduced this lemma from a proof of the hyperbolic KAM theorem, considering the first iteration in the KAM iterative process. Nevertheless, since we do not give in this paper the proof of this theorem, we have included here a direct proof of lemma 2.

2. Proceeding analogously to this lemma, one can easily obtain the following generalization: for any given first integral $F$ of $H_{0}$, one has the approximation $F\left(z^{*}(\varphi)\right)-\overline{F\left(z^{*}(\cdot)\right)}=\mu\{F, \chi\}\left(z_{0}^{*}(\varphi)\right)+$ $\mathrm{O}\left(\mu^{2}\right)$.

3. The mean value $\overline{I^{*}}$ is closely related to the constant $a$ in theorem 1 . Using the exactness of the normalizing transformation $\Phi$, one can check that $\overline{I^{*}}-a=\mathrm{O}\left(\mu^{2}\right)$. However, we will not need this fact later.

For the local perturbed whiskers $\mathcal{W}_{\text {loc }}^{ \pm}$, it is easy to establish that

$$
\lim _{s \rightarrow \pm \infty}\left[z_{\text {loc }}^{ \pm}(s, \varphi)-z^{*}(\varphi \pm \pi \lambda)\right]=0
$$

like in (12), as well as exponentially decreasing bounds like in (13), but replacing the Lyapunov exponents $\pm \alpha$ by $\pm b \alpha$. More precisely, the following lemma provides an asymptotic formula for the local whiskers $\mathcal{W}_{\text {loc }}^{ \pm}$near the torus $\mathcal{T}$, to be used in section 3.1. 
Lemma 3 For any $\pm s \geq s_{0}$ and $\varphi \in \mathbb{T}^{n}$, one has

$$
z_{\mathrm{loc}}^{ \pm}(s, \varphi)=z^{*}(\varphi \pm \pi \lambda)+z_{0}(s, \mp \pi \lambda)+\mathrm{O}\left(e^{\mp \alpha s / 2} \mu\right) .
$$

Proof. We proceed simultaneously for the local stable whisker and the unstable one. Denoting $\Phi^{(1)}=$ $\Phi-$ id, we have

$$
z_{\mathrm{loc}}^{-}(s, \varphi)-z^{*}(\varphi \pm \pi \lambda)=\tilde{z}(s, \varphi)-\tilde{z}^{*}(\varphi \pm \pi \lambda)+\Phi^{(1)}(\tilde{z}(s, \varphi))-\Phi^{(1)}\left(\tilde{z}^{*}(\varphi \pm \pi \lambda)\right) .
$$

From (18), we note that

$$
\tilde{z}(s, \varphi)-\tilde{z}^{*}(\varphi \pm \pi \lambda)=z_{0}(b s, \mp \pi \lambda)
$$

(which does not depend on $\varphi$ ). We have the inequality

$$
\left|z_{0}(b s, \mp \pi \lambda)-z_{0}(s, \mp \pi \lambda)\right| \leq\left|\partial_{s} z_{0}\right||(b-1) s| \leq C e^{\mp \alpha s / 2}|\mu| .
$$

To obtain this, we have used the fact that $\partial_{s} z_{0}(s, \varphi)=\left(y_{0}(s),-V^{\prime}\left(x_{0}(s)\right), y_{0}(s) \lambda, 0\right)$ is exponentially decreasing to 0 for $s \rightarrow \pm \infty$, together with a bound of the type $|s| e^{\mp s} \leq e^{\mp s / 2}$, and also that we can assume $|b-1| \leq 1 / 2$ from (17). On the other hand, we have the following inequality:

$$
\left|\Phi^{(1)}(\tilde{z}(s, \varphi))-\Phi^{(1)}\left(\tilde{z}^{*}(\varphi \pm \pi \lambda)\right)\right| \leq\left|\mathrm{d} \Phi^{(1)}\right|\left|\tilde{z}(s, \varphi)-\tilde{z}^{*}(\varphi \pm \pi \lambda)\right| \leq C^{\prime}|\mu| C^{\prime \prime} e^{\mp b \alpha s} .
$$

Here, we have obtained from (17) a bound for $\left|\mathrm{d} \Phi^{(1)}\right|$ (on a real domain), and we have also used a bound analogous to (13), with $\mp b \alpha$ instead of $\mp \alpha$. By (17), we can assume $b \geq 1 / 2$. Then the proof is finished by combining the two exponential bounds obtained.

\subsection{Parameterization of the perturbed global whiskers}

The parameterizations of the whiskers $\mathcal{W}_{\text {loc }}^{ \pm}$, introduced in section 2.2 , valid for $\pm s \geq s_{0}$, can easily be extended to further values of $s$ in a natural way, since the whiskers are formed by trajectories associated to our Hamiltonian $H$. We denote $\mathcal{W}^{ \pm}$the extended or global whiskers; our aim is to measure the distance between them.

We will denote $\Upsilon^{t}$ and $\Upsilon_{0}^{t}$ the time- $t$ Hamiltonian flows associated to $H$ and $H_{0}$ respectively, for real values of $t$.

The extended parameterizations $z^{ \pm}(s, \varphi)$ for the global whiskers are defined in such a way that the trajectories on $\mathcal{W}^{ \pm}$are given by $t \mapsto z^{ \pm}(s+t, \varphi+\omega t)$. For the global unstable whisker we define, as long as the domain of $H$ is not left,

$$
z^{-}(s, \varphi)=\Upsilon^{s-\hat{s}}\left(z_{\mathrm{loc}}^{-}(\hat{s}, \varphi-\omega(s-\hat{s}))\right),
$$

with any $\hat{s}<-s_{0}$. From the properties of the flow, we see that this definition does not depend on the choice of $\hat{s}$. Note that the analyticity of $z^{-}(s, \varphi)$ with respect to $s, \varphi$ can be established in the following simple way: for any $s$ in a neighborhood of some fixed $s^{*}$, we choose $\hat{s}=s-\left(s^{*}-\hat{s}^{*}\right)$ (analytic in $s$ ); then one has

$$
z^{-}(s, \varphi)=\Upsilon^{s^{*}-\hat{s}^{*}}\left(z_{\text {loc }}^{-}\left(s-\left(s^{*}-\hat{s}^{*}\right), \varphi-\omega\left(s^{*}-\hat{s}^{*}\right)\right)\right),
$$

which is clearly analytic in $s, \varphi$. Proceeding similarly, we can define $z^{+}(s, \varphi)$ for the global stable whisker.

We assume our starting Hamiltonian $H$ in (1) analytic on a complex domain

$$
D_{r}=\{(x, y, \varphi, I):|\operatorname{Im} x|,|y|,|\operatorname{Im} \varphi|,|I| \leq r\},
$$

with $r>0$. Note that $D_{r} \supset B_{r}$, but the new domain $D_{r}$ is global in Re $x \in \mathbb{T}$. We will assume $r$ big enough in order to contain a neighborhood of the (global) unperturbed whiskers: say $|y| \leq r / 2$ on $\mathcal{W}_{0}$. As we see below, the domain $D_{r}$ can also be ensured to contain a large piece of the perturbed global whiskers $\mathcal{W}^{ \pm}$. 
It is not hard to establish, from the variational equations for $H_{0}$, the following inequalities: for given $z, z^{\prime}$

$$
\left|\Upsilon_{0}^{t}\left(z^{\prime}\right)-\Upsilon_{0}^{t}(z)\right| \leq e^{K_{1}|t|}\left|z^{\prime}-z\right|, \quad\left|\Upsilon^{t}(z)-\Upsilon_{0}^{t}(z)\right| \leq K_{2}\left(e^{K_{1}|t|}-1\right)|\mu|,
$$

for any $t$ as long as the flows remain in the domain of $H$. The constants $K_{j}$ do not depend on $\omega, \mu$. Note that the factor $K_{2}|\mu|$ in the second inequality comes from the fact that $\nabla\left(H-H_{0}\right)=\mathrm{O}(\mu)$.

It can be deduced from the inequalities (29) that a trajectory $z(t)$ with initial condition $z\left(t_{1}\right)$ at distance $\mathrm{O}(\mu)$ to the unperturbed whisker $\mathcal{W}_{0}$ remains at distance $\mathrm{O}(\mu)$ for a time interval $\mathrm{O}(1)$. Then the perturbed global whiskers $\mathcal{W}^{ \pm}$remain close to $\mathcal{W}_{0}$, for an interval of values of the parameter $s$. The following lemma gives a more precise statement of this property. We recall that we are dealing here only with real trajectories. Similar estimates for complex trajectories are more involved, and require to apply an extension theorem, like in [DS97, DGJS97, RW98].

The lemma refers only to the unstable whisker $\mathcal{W}^{-}$. The version for the stable whisker $\mathcal{W}^{+}$is completely analogous.

Lemma 4 The bound

$$
z^{-}(s, \varphi)=z_{0}(s, \varphi)+\mathrm{O}\left(e^{K_{1} s} \mu\right)
$$

holds for any $\varphi \in \mathbb{T}^{n}$ and for any real s such that

$$
s \geq-s_{0}, \quad e^{K_{1} s}|\mu| \leq \frac{1}{C}
$$

and then $z^{-}(s, \varphi)$ remains in $D_{r}$.

Proof. For given $s \geq-s_{0}$ and $\varphi$, let $-2 s_{0}<\hat{s}<-s_{0}$, and $\hat{\varphi}=\varphi-\omega(s-\hat{s})$. Using (17), we can easily establish the inequality

$$
\left|z_{\text {loc }}^{-}(\hat{s}, \hat{\varphi})-z_{0}(\hat{s}, \hat{\varphi})\right| \leq C^{\prime}|\mu| \text {. }
$$

Then applying (29), we obtain:

$$
\begin{aligned}
\left|z^{-}(s, \varphi)-z_{0}(s, \varphi)\right| & =\left|\Upsilon^{s-\hat{s}}\left(z_{\text {loc }}^{-}(\hat{s}, \hat{\varphi})\right)-\Upsilon_{0}^{s-\hat{s}}\left(z_{0}(\hat{s}, \hat{\varphi})\right)\right| \\
& \leq\left|\Upsilon^{s-\hat{s}}\left(z_{\text {loc }}^{-}(\hat{s}, \hat{\varphi})\right)-\Upsilon_{0}^{s-\hat{s}}\left(z_{\text {loc }}^{-}(\hat{s}, \hat{\varphi})\right)\right|+\left|\Upsilon_{0}^{s-\hat{s}}\left(z_{\text {loc }}^{-}(\hat{s}, \hat{\varphi})\right)-\Upsilon_{0}^{s-\hat{s}}\left(z_{0}(\hat{s}, \hat{\varphi})\right)\right| \\
& \leq K_{2}\left(e^{K_{1}(s-\hat{s})}-1\right)|\mu|+e^{K_{1}(s-\hat{s})}\left|z_{\text {loc }}^{-}(\hat{s}, \hat{\varphi})-z_{0}(\hat{s}, \hat{\varphi})\right| \\
& \leq C^{\prime \prime} e^{K_{1} s}|\mu| \leq \frac{C^{\prime \prime}}{C}=\frac{r}{2},
\end{aligned}
$$

provided we choose $C=2 C^{\prime \prime} / r$ in (30). In this way, we keep $z^{-}(s, \varphi)$ in $D_{r}$.

\section{The Poincaré-Melnikov method for whiskered tori}

\subsection{Melnikov potential and Melnikov function}

In order to provide a first order approximation for the splitting, we introduce the (scalar) Melnikov potential and the (vector) Melnikov function as, respectively, the following functions, $\mu$-independent and periodic in $\varphi \in \mathbb{T}^{n}$ :

$$
\begin{aligned}
L(\varphi) & =-\int_{-\infty}^{\infty}\left(H_{1}-\overline{H_{1}}-\left\{\chi, H_{0}\right\}\right)\left(z_{0}(t, \varphi+\omega t)\right) \mathrm{d} t \\
M(\varphi) & =\partial_{\varphi} L(\varphi)=-\int_{-\infty}^{\infty}\left[\partial_{\varphi}\left(H_{1}-\left\{\chi, H_{0}\right\}\right)\right]\left(z_{0}(t, \varphi+\omega t)\right) \mathrm{d} t .
\end{aligned}
$$

Recall that $\overline{H_{1}}(x, y, I)$ denotes the $\varphi$-average of $H_{1}$, and that $\chi(x, y, \varphi, I)=\chi(\varphi)$ is the (zero average) function solving the small divisors equation (19). Notice that $\bar{L}=0$ (and of course $\bar{M}=0$ ), because the function inside the integral has zero average. 
We point out that the Melnikov integrals introduced in (31-32) are absolutely convergent. Indeed, note that the function $H_{1}-\overline{H_{1}}-\left\{\chi, H_{0}\right\}$ vanishes on $\mathcal{T}_{0}$ :

$$
\left(H_{1}-\overline{H_{1}}-\left\{\chi, H_{0}\right\}\right)\left(z_{0}^{*}(\varphi)\right)=\left(H_{1}-\overline{H_{1}}-\left\langle\omega, \partial_{\varphi} \chi\right\rangle\right)\left(z_{0}^{*}(\varphi)\right)=0 \quad \forall \varphi \in \mathbb{T}^{n} .
$$

Using this fact, together with the exponentially decreasing bounds (13), we obtain the absolute convergence. We recall that the function $\chi$, which gives the absolute convergence, is closely related to the shift of the perturbed torus $\mathcal{T}$ with respect to the unperturbed torus $\mathcal{T}_{0}$ in applying KAM theorem, as shown in lemma 2. Our use of absolutely convergent integrals in the formulation of the Poincaré-Melnikov method for whiskered tori makes a difference with respect to some previous works [HM82, Wig88, Rob88], where conditionally convergent integrals are used and the integration limits have to be carefully chosen.

Another important fact is that our formulas for $L$ and $M$ are useful in both the coupled and the uncoupled cases (in (2), $\lambda \neq 0$ and $\lambda=0$ respectively), as we illustrate in sections 3.2 and 3.3. Related expressions, also valid in both cases, were previously obtained by Treschev [Tre94]. In that paper, the Melnikov function was expressed with the help of some correcting terms giving rise to the absolute convergence. We have improved that expression, obtaining a more compact formula (32), which includes the correcting terms in the integral (the Melnikov potential was not introduced in [Tre94]).

It is useful to provide alternative expressions for the Melnikov potential and the Melnikov function, similar to the ones given in [Tre94]. These expressions contain the correcting terms quoted just above:

$$
\begin{aligned}
L(\varphi) & =\lim _{T \rightarrow \infty}\left[-\int_{-T}^{T}\left(H_{1}-\overline{H_{1}}\right)\left(z_{0}(t, \varphi+\omega t)\right) \mathrm{d} t+\chi(\varphi+\pi \lambda+\omega T)-\chi(\varphi-\pi \lambda-\omega T)\right], \\
M(\varphi) & =\lim _{T \rightarrow \infty}\left[-\int_{-T}^{T} \partial_{\varphi} H_{1}\left(z_{0}(t, \varphi+\omega t)\right) \mathrm{d} t+\partial_{\varphi} \chi(\varphi+\pi \lambda+\omega T)-\partial_{\varphi} \chi(\varphi-\pi \lambda-\omega T)\right] .
\end{aligned}
$$

To show that (31) is equal to (34), note that

$$
\int_{-T}^{T}\left\{\chi, H_{0}\right\}\left(z_{0}(t, \varphi+\omega t)\right) \mathrm{d} t=\int_{-T}^{T} \frac{\mathrm{d}}{\mathrm{d} t} \chi\left(z_{0}(t, \varphi+\omega t)\right) \mathrm{d} t=\chi\left(z_{0}(T, \varphi+\omega T)\right)-\chi\left(z_{0}(-T, \varphi-\omega T)\right)
$$

and, in view of (12), this expression inside the limit in (31) can be substituted by $\chi(\varphi+\pi \lambda+\omega T)-$ $\chi(\varphi-\pi \lambda-\omega T)$. This gives (34), and we obtain (35) just taking a $\varphi$-derivative.

We recall also that an expression equivalent to our Melnikov potential, valid in the coupled case, appeared already in [CG94, §4] in terms of its Fourier expansion. However, our expression (31) looks much simpler.

Concerning the uncoupled case $\lambda=0$, we can obtain a simpler and perhaps more classical formula for the Melnikov potential (and, obviously, for the Melnikov function):

$$
\begin{aligned}
L(\varphi) & =-\int_{-\infty}^{\infty}\left[\left(H_{1}-\overline{H_{1}}\right)\left(z_{0}(t, \varphi+\omega t)\right)-\left(H_{1}-\overline{H_{1}}\right)\left(z_{0}^{*}(\varphi+\omega t)\right)\right] \mathrm{d} t \\
& =-\int_{-\infty}^{\infty}\left[H_{1}\left(z_{0}(t, \varphi+\omega t)\right)-H_{1}\left(z_{0}^{*}(\varphi+\omega t)\right)\right] \mathrm{d} t+\mathrm{const}
\end{aligned}
$$

as one deduces from the following computation, valid for any $t_{1}, t_{2}$ :

$$
\begin{aligned}
\chi\left(\varphi+\omega t_{2}\right)-\chi\left(\varphi+\omega t_{1}\right)=\int_{t_{1}}^{t_{2}} \frac{\mathrm{d}}{\mathrm{d} t} \chi(\varphi+\omega t) \mathrm{d} t \\
\quad=\int_{t_{1}}^{t_{2}}\left\{\chi, H_{0}\right\}\left(z_{0}^{*}(\varphi+\omega t)\right) \mathrm{d} t=\int_{t_{1}}^{t_{2}}\left(H_{1}-\overline{H_{1}}\right)\left(z_{0}^{*}(\varphi+\omega t)\right) \mathrm{d} t,
\end{aligned}
$$

where we have used (33).

Another simple case is that of a perturbation vanishing on the whiskered torus, $H_{1}=\mathrm{O}_{2}(x, y, I)$. In this case, the whiskered torus remains unchanged. We have $\chi=0$ in (31) and therefore the following integral is absolutely convergent and can be applied:

$$
L(\varphi)=-\int_{-\infty}^{\infty}\left(H_{1}-\overline{H_{1}}\right)\left(z_{0}(t, \varphi+\omega t)\right) \mathrm{d} t=-\int_{-\infty}^{\infty} H_{1}\left(z_{0}(t, \varphi+\omega t)\right) \mathrm{d} t+\text { const. }
$$


The absolute convergence of the integral for the Melnikov function in this case was already pointed out in [Rob88]. For $n=1,(37)$ coincides with [DR97, formula (2.15)].

Coming again to the general case considered at the beginning, the following standard result shows that a first order approximation for the splitting between the global whiskers is given in terms of the Melnikov function $M$. Since both whiskers are $(n+1)$-dimensional manifolds contained in the same $(2 n+1)$-dimensional level of energy, it is enough to express its distance by an $n$-dimensional measure. We take the difference $I^{-}-I^{+}$as the measure for the splitting (we denote $I^{ \pm}(s, \varphi)$ the $I$-component of the parameterizations $\left.z^{ \pm}(s, \varphi)\right)$.

Proposition 5 Assuming $|\mu|$ small enough, one has for any $|s| \leq s_{0}$ and $\varphi \in \mathbb{T}^{n}$ the following approximation:

$$
I^{-}(s, \varphi)-I^{+}(s, \varphi)=\mu \partial_{\varphi} L(\varphi-\omega s)+\mathrm{O}\left(\mu^{2}\right) .
$$

Proof. For $e^{K_{1} s_{0}}|\mu| \leq 1 / C$, we can apply lemma 4 directly and then $I^{-}(s, \varphi)$ and $I^{+}(s, \varphi)$ can both be defined in the whole interval $-s_{0} \leq s \leq s_{0}$. We write

$$
\begin{aligned}
I^{-}(s, \varphi)-I^{+}(s, \varphi)= & \left(I^{-}(s, \varphi)-I^{*}(\varphi-\pi \lambda)\right)-\left(I^{+}(s, \varphi)-I^{*}(\varphi+\pi \lambda)\right) \\
& +\left(I^{*}(\varphi-\pi \lambda)-I^{*}(\varphi+\pi \lambda)\right) .
\end{aligned}
$$

First, we are going to write the first two terms in this expression as integrals. Applying that $\dot{I}=$ $-\partial_{\varphi} H=-\mu \partial_{\varphi} H_{1}$ (so we are using that $I_{1}, \ldots, I_{n}$ are first integrals of the unperturbed Hamiltonian $\left.H_{0}\right)$, we obtain:

$$
\begin{aligned}
& I^{ \pm}(s, \varphi)-I^{*}(\varphi \pm \pi \lambda) \\
& \quad=-\int_{s}^{ \pm \infty} \frac{\mathrm{d}}{\mathrm{d} t}\left[I^{ \pm}(t, \varphi-\omega s+\omega t)-I^{*}(\varphi-\omega s \pm \pi \lambda+\omega t)\right] \mathrm{d} t \\
& \quad=\mu \int_{s}^{ \pm \infty}\left[\partial_{\varphi} H_{1}\left(z^{ \pm}(t, \varphi-\omega s+\omega t)\right)-\partial_{\varphi} H_{1}\left(z^{*}(\varphi-\omega s \pm \pi \lambda+\omega t)\right)\right] \mathrm{d} t .
\end{aligned}
$$

Note that lemma 3 ensures the absolute convergence of these improper integrals.

For the first order approximations of the parameterizations involved, write

$$
I^{ \pm}(s, \varphi)=\mu I_{1}^{ \pm}(s, \varphi)+\mathrm{O}\left(\mu^{2}\right), \quad I^{*}(\varphi)=\mu I_{1}^{*}(\varphi)+\mathrm{O}\left(\mu^{2}\right),
$$

and recall from lemma 2 that $I_{1}^{*}(\varphi)=\zeta-\partial_{\varphi} \chi(\varphi)$. It is clear that

$$
\begin{aligned}
I_{1}^{-}(s, \varphi)-I_{1}^{+}(s, \varphi)= & \left(I_{1}^{-}(s, \varphi)-I_{1}^{*}(\varphi-\pi \lambda)\right)-\left(I_{1}^{+}(s, \varphi)-I_{1}^{*}(\varphi+\pi \lambda)\right) \\
& +\left(I_{1}^{*}(\varphi-\pi \lambda)-I_{1}^{*}(\varphi+\pi \lambda)\right) .
\end{aligned}
$$

Considering the first order in $\mu$ in (39), we get the following integrals, along unperturbed homoclinic trajectories:

$$
\begin{aligned}
I_{1}^{ \pm}(s, \varphi)-I_{1}^{*}(\varphi \pm \pi \lambda)= & \int_{s}^{ \pm \infty}\left[\partial_{\varphi} H_{1}\left(z_{0}(t, \varphi-\omega s+\omega t)\right)-\partial_{\varphi} H_{1}\left(z_{0}^{*}(\varphi-\omega s \pm \pi \lambda+\omega t)\right)\right] \mathrm{d} t \\
= & \lim _{T \rightarrow \infty}\left[\int_{s}^{ \pm T} \partial_{\varphi} H_{1}\left(z_{0}(t, \varphi-\omega s+\omega t)\right) \mathrm{d} t\right. \\
& \left.\quad-\partial_{\varphi} \chi(\varphi-\omega s \pm \pi \lambda \pm \omega T)+\partial_{\varphi} \chi(\varphi \pm \pi \lambda)\right]
\end{aligned}
$$

where we have used the equality (36), rewriting there $\varphi$ as $\varphi-\omega s \pm \pi \lambda$ and taking a $\varphi$-derivative. Using also that

$$
I_{1}^{*}(\varphi-\pi \lambda)-I_{1}^{*}(\varphi+\pi \lambda)=-\partial_{\varphi} \chi(\varphi-\pi \lambda)+\partial_{\varphi} \chi(\varphi+\pi \lambda)
$$

we obtain the first order approximation of (38):

$$
I_{1}^{-}(s, \varphi)-I_{1}^{+}(s, \varphi)=M(\varphi-\omega s)=\partial_{\varphi} L(\varphi-\omega s),
$$


with the function $M$ according to the expression (35), which is equivalent to our definition (32).

To end the proof, we still have to bound the remainder and show that the constant in the $\mathrm{O}\left(\mu^{2}\right)$-term in (38) does not depend on $\omega$. First, we consider the difference between (39) and its approximation given by $(40)$ :

$$
\begin{aligned}
& \left(I^{ \pm}(s, \varphi)-I^{*}(\varphi \pm \pi \lambda)\right)-\mu\left(I_{1}^{ \pm}(s, \varphi)-I_{1}^{*}(\varphi \pm \pi \lambda)\right) \\
& =\mu \int_{s}^{ \pm \infty}\left[\partial_{\varphi} H_{1}\left(z^{ \pm}(t, \varphi-\omega s+\omega t)\right)-\partial_{\varphi} H_{1}\left(z^{*}(\varphi-\omega s \pm \pi \lambda+\omega t)\right)\right. \\
& \left.\quad-\partial_{\varphi} H_{1}\left(z_{0}(t, \varphi-\omega s+\omega t)\right)+\partial_{\varphi} H_{1}\left(z_{0}^{*}(\varphi-\omega s \pm \pi \lambda+\omega t)\right)\right] \mathrm{d} t \\
& \quad=\mathrm{O}\left(\mu^{2}\right) .
\end{aligned}
$$

To obtain this estimate, the integral has been broken in two parts: $\int_{ \pm s_{0}}^{ \pm \infty}$ and $\int_{s}^{ \pm s_{0}}$. For the first part, we have used the exponentially decreasing estimate of lemma 3 and, with the help of the mean value theorem, we have obtained an $\mathrm{O}\left(\mu^{2}\right)$-estimate. For the second part of the integral, we have used the extended estimate of lemma 4 and another simpler estimate to obtain also an $\mathrm{O}\left(\mu^{2}\right)$-estimate. Note that, for this second part, the length of the interval of the integral can be bounded by a constant: $\left| \pm s_{0}-s\right| \leq 2 s_{0}$. Finally, it follows from lemma 2 the following estimate for the last term:

$$
\left(I^{*}(\varphi-\pi \lambda)-I^{*}(\varphi+\pi \lambda)\right)-\mu\left(I_{1}^{*}(\varphi-\pi \lambda)-I_{1}^{*}(\varphi+\pi \lambda)\right)=\mathrm{O}\left(\mu^{2}\right) .
$$

Combining these estimates, we obtain (38).

Remark. The approximation for $I^{-}(s, \varphi)-I^{+}(s, \varphi)$ only depends on $\varphi-\omega s$ at first order in $\mu$ but, in general, it has a more complicated dependence at higher orders. In the same way, the first order approximation is a gradient of a scalar function $L$ but we cannot ensure this fact at higher orders.

As a simple corollary of proposition 5, we see that in the regular case the simple zeros of the Melnikov function $M$ give rise, for $|\mu|$ small enough, to transverse homoclinic intersections between the perturbed whiskers. As is well-known, if a point belongs to the homoclinic intersection, then its whole orbit is also contained in the intersection (this fact is closely related to the dependence on $\varphi-\omega s$ ). Thus, it is enough to find the zeros of $M(\varphi-\omega s)$ for a fixed value of $s$ (a $\varphi$-section), and from the simple zeros of $M$ we get transverse homoclinic orbits biasymptotic to the perturbed torus (contained in both the stable and the unstable whiskers).

Since the function $M$ is the gradient of the Melnikov potential $L$, it is obvious that the simple zeros of $M$ are the nondegenerate critical points of $L$. If the function $L$ (defined on $\mathbb{T}^{n}$ ) is a Morse function (its critical points are all nondegenerate: a generic property), we deduce from Morse theory that for $|\mu|$ small enough there exist at least $2^{n}$ transverse homoclinic orbits.

It is well-known that this argument does not apply in the singular case, $\omega=\omega^{*} / \sqrt{\varepsilon}$ and $\mu=\varepsilon^{p}$, because the Melnikov function $M$ is typically exponentially small in $\varepsilon$ (see the example in section 3.3). To ensure that $\mu M(\varphi-\omega s)$ dominates the $\mathrm{O}\left(\mu^{2}\right)$-term, one has to assume $\mu$ exponentially small with respect to $\varepsilon$. For larger values of $\mu$, the existence of intersections cannot follow directly from (38).

As said in the introduction, the study of the splitting in the singular case requires a more careful analysis, which is not carried out in this paper (see instead [DGJS97, RW98, GGM99]). Nevertheless, the effective existence of a number of homoclinic intersections, for both the regular and singular cases, will be established in section 5.2. This will require to introduce other variables in which the difference $I^{-}-I^{+}$(and not only its first order approximation) becomes the gradient with respect to the angle variables of some periodic function, called splitting potential.

Another important fact is that, in the proof of proposition 5, we have used that the $n$ components of $I$ are first integrals of $H_{0}$. Following Treschev [Tre94], we can generalize proposition 5 in order to give an analogous first order approximation for the difference $F\left(z^{-}(s, \varphi)\right)-F\left(z^{+}(s, \varphi)\right)$, where $F$ is any given first integral of the unperturbed Hamiltonian $H_{0}$. Define the (scalar) function

$$
M_{F}(\varphi)=\int_{-\infty}^{\infty}\left\{F, H_{1}-\left\{\chi, H_{0}\right\}\right\}\left(z_{0}(t, \varphi+\omega t)\right) \mathrm{d} t
$$


(again, this formula is more compact than in [Tre94]). In the uncoupled case $(\lambda=0)$, this function can also be written as

$$
M_{F}(\varphi)=\int_{-\infty}^{\infty}\left[\left\{F, H_{1}\right\}\left(z_{0}(t, \varphi+\omega t)\right)-\left\{F, H_{1}\right\}\left(z_{0}^{*}(\varphi+\omega t)\right)\right] \mathrm{d} t .
$$

The difference between the values of the first integral $F$ on both manifolds admits the following first order approximation:

$$
F\left(z^{-}(s, \varphi)\right)-F\left(z^{+}(s, \varphi)\right)=\mu M_{F}(\varphi-\omega s)+\mathrm{O}\left(\mu^{2}\right) .
$$

We omit the proof because it is analogous to that of proposition 5 .

Let us consider now some interesting particular cases. Taking $F=I_{j}$, it is clear that

$$
M_{I_{j}}(\varphi)=\partial_{\varphi_{j}} L(\varphi), \quad j=1, \ldots, n
$$

For $F=H_{0}$, it is not hard to see from (41) that $M_{H_{0}}=0$. Taking into account that the function $\widehat{P}$ defined in (6) is also a first integral of $H_{0}$, we deduce from (8) that, considering $F=\widehat{P}$,

$$
M_{\widehat{P}}(\varphi-\omega s)=-\left\langle\omega, \partial_{\varphi} L(\varphi-\omega s)\right\rangle=\frac{\mathrm{d}}{\mathrm{d} s} L(\varphi-\omega s) .
$$

Thus, the vector $\left(M_{\widehat{P}}, M_{I_{1}}, \ldots, M_{I_{n}}\right)$ is the gradient of the Melnikov potential $L(\varphi-\omega s)$ when considered a function of all the variables $s, \varphi$ (i.e. a function defined on the separatrix $\mathcal{W}_{0}$ ).

\subsection{A computable coupled example}

Now we illustrate the computation of the Melnikov integrals in a simple coupled case, with $n+1$ degrees of freedom. Afterwards, we consider a more particular case and show (in the regular case) the existence of a number of transverse homoclinic intersections.

Let us introduce our example $H=H_{0}+\mu H_{1}$. For the integrable part, we choose the standard pendulum $V(x)=\cos x-1$, and include a coupling term with $\lambda \neq 0$ :

$$
H_{0}=\langle\omega, I\rangle+\frac{1}{2}\langle\Lambda I, I\rangle+\frac{y^{2}}{2}+\cos x-1+\langle\lambda, I\rangle y .
$$

In the perturbation, we consider a real function $H_{1}$ only depending on $\varphi$ :

$$
H_{1}(\varphi)=\sum_{k \in \mathbb{Z}^{n}} h_{k} e^{i\langle k, \varphi\rangle} .
$$

It is important to notice that for $\lambda=0$, the Hamiltonian $H$ decouples, and there is no possibility of splitting of the separatrices of $H_{0}$. So we assume $\lambda \neq 0$. With this perturbation $H_{1}(\varphi)$, the solution $\chi(\varphi)$ of equation (19) is simply

$$
\chi(\varphi)=\sum_{k \neq 0} \frac{h_{k}}{i\langle k, \omega\rangle} e^{i\langle k, \varphi\rangle}
$$

and the Melnikov potential is given by its Fourier series $L(\varphi)=\sum_{k \neq 0} L_{k} e^{i\langle k, \varphi\rangle}$, where each coefficient can be written as $L_{k}=h_{k} l_{k}$, where

$$
l_{k}=\frac{\langle k, \lambda\rangle}{\langle k, \omega\rangle} \mathcal{J}(\langle k, \omega\rangle,\langle k, 2 \lambda\rangle),
$$

and we define

$$
\mathcal{J}(a, b)=\int_{-\infty}^{\infty} e^{i a t} e^{i b\left(x_{0}(t)-\pi\right) / 2} y_{0}(t) \mathrm{d} t
$$


which is always real provided $a, b$ are real (we integrate an odd function in the imaginary part). In this integral, $\left(x_{0}(t), y_{0}(t)\right)$ is the well-known (positive) homoclinic trajectory of the standard pendulum:

$$
x_{0}(t)=4 \arctan e^{t}, \quad y_{0}(t)=\dot{x}_{0}(t)=\frac{2}{\cosh t} .
$$

For the sake of simplicity, we will assume that $\lambda$ is "half-integer" (i.e. $2 \lambda \in \mathbb{Z}^{n}$ ). In this way, we only have to consider integer values of $b=\langle k, 2 \lambda\rangle$ in the integral (43), which can computed in this case using residue theory. Indeed, one has

$$
e^{i\left(x_{0}(t)-\pi\right) / 2}=\frac{1+i \sinh t}{\cosh t}
$$

and hence the function inside the integral (43) has only one singularity in the complex domain $0 \leq$ $\operatorname{Im} t \leq 2 \pi$ : a pole of order $b+1$ at $t=3 \pi i / 2$ (this singularity becomes logarithmic if $b$ is not integer, and then residue theory cannot be applied). Some computations from (45) lead for $b \geq 0$ to the following formula:

$$
\begin{aligned}
\mathcal{J}(a, b)= & \sum_{0 \leq p \leq l \leq b / 2} 2(-1)^{p}\left(\begin{array}{c}
b \\
2 l
\end{array}\right)\left(\begin{array}{l}
l \\
p
\end{array}\right) \mathcal{I}(a, b-2 p+1) \\
& +\sum_{0 \leq p \leq l \leq(b-1) / 2} 2(-1)^{p+1}\left(\begin{array}{c}
b \\
2 l+1
\end{array}\right)\left(\begin{array}{l}
l \\
p
\end{array}\right) \frac{a}{b-2 p} \mathcal{I}(a, b-2 p),
\end{aligned}
$$

where we define

$$
\mathcal{I}(a, r)=\int_{-\infty}^{\infty} \frac{\cos a t}{\cosh ^{r} t} \mathrm{~d} t
$$

This integral satisfies the recurrence

$$
\mathcal{I}(a, r)=\frac{a^{2}+(r-2)^{2}}{(r-1)(r-2)} \mathcal{I}(a, r-2),
$$

and can be computed from

$$
\mathcal{I}(a, 1)=\frac{\pi}{\cosh (\pi a / 2)}, \quad \mathcal{I}(a, 2)=\frac{\pi a}{\sinh (\pi a / 2)},
$$

which are computed by residue theory. Thus, we are able to compute $\mathcal{J}(a, b)$ for any real $a$ and integer $b \geq 0$. (For any real $b \geq 0$ but not integer, we can still express $\mathcal{J}(a, b)$ in terms of $\mathcal{I}(a, b-[b]$ ) and $\mathcal{I}(a, b-[b]+1)$, where $[b]$ denotes the integer part of $b$.) For integer $b<0$, we can use the equality $\mathcal{J}(a, b)=\mathcal{J}(-a,-b)$. For instance, we obtain

$$
\mathcal{J}(a, 0)=\frac{2 \pi}{\cosh (\pi a / 2)}, \quad \mathcal{J}(a, \pm 1)=\frac{4 \pi a e^{\mp \pi a / 2}}{\sinh \pi a}, \quad \mathcal{J}(a, \pm 2)=\mp \frac{4 \pi a^{2} e^{\mp \pi a / 2}}{\sinh \pi a} .
$$

Next, in order to show the existence of transverse homoclinic intersections, we consider a more specific example, with a finite number of harmonics. To obtain this result, the perturbation $H_{1}(\varphi)$ requires at least $n$ harmonics (as it will be clear below). So we consider a trigonometric polynomial

$$
H_{1}(\varphi)=\sum_{j=1}^{n} 2\left|h_{j}\right| \cos \left(\left\langle k^{(j)}, \varphi\right\rangle+\eta_{j}\right)
$$

(for simplicity, we write $h_{j}$ instead of $h_{k^{(j)}}$ ). We assume that the coefficients $h_{j}$ are all nonvanishing, and that the integer vectors $k^{(j)}$ are linearly independent; denote $\Delta=\operatorname{det}\left(k^{(1)}, \ldots, k^{(n)}\right) \neq 0$. If the factors $l_{j}\left(=l_{k^{(j)}}\right)$, obtained from (42), are all nonvanishing, we are going to show that the Melnikov potential $L(\varphi)$ associated to this problem is a Morse function having exactly $2^{n}|\Delta|$ critical points. For instance, a concrete example in which this result applies is $\lambda=\frac{1}{2} e_{1}, k^{(1)}=e_{1}, k^{(j)}=e_{1}+e_{j}, j=2, \ldots, n$ (where $e_{1}, \ldots, e_{n}$ denotes the canonical basis of $\left.\mathbb{Z}^{n}\right)$; in this case we have $l_{j} \neq 0$ and $\Delta=1$, and therefore exactly $2^{n}$ critical points. 
Writing the trigonometric polynomial $H_{1}(\varphi)$ in the exponential form and using that $l_{k}=l_{-k}$ in (42), we obtain

$$
L(\varphi)=\sum_{j=1}^{n} 2\left|h_{j}\right| l_{j} \cos \left(\left\langle k^{(j)}, \varphi\right\rangle+\eta_{j}\right)
$$

Then we have

$$
M(\varphi)=-\sum_{j=1}^{n} 2 k^{(j)}\left|h_{j}\right| l_{j} \sin \left(\left\langle k^{(j)}, \varphi\right\rangle+\eta_{j}\right),
$$

and therefore the critical points are the solutions $\varphi^{*}$ of the following $2^{n}$ linear systems on $\mathbb{T}^{n}$, where each $\alpha_{j}$ can be 0 or $\pi$ :

$$
\left\langle k^{(j)}, \varphi^{*}\right\rangle+\eta_{j}=\alpha_{j} \quad(\bmod 2 \pi), \quad j=1, \ldots, n
$$

(notice that the critical points obtained are exactly those of $H_{1}(\varphi)$ ). For each choice of $\left(\alpha_{1}, \ldots, \alpha_{n}\right)$, the system has $|\Delta|$ solutions (see for instance [LM88, appendix 3]); so the number of critical points is $2^{n}|\Delta|$. To see that any critical point is nondegenerate, we compute the determinant of the symmetric matrix $\partial_{\varphi} M\left(\varphi^{*}\right)=\partial_{\varphi}^{2} L\left(\varphi^{*}\right)$, which gives also a measure of the transversality:

$$
\begin{aligned}
\operatorname{det} \partial_{\varphi}^{2} L\left(\varphi^{*}\right) & =\operatorname{det}\left(-2 \sum_{j=1}^{n} k^{(j)}\left(k^{(j)}\right)^{\top}\left|h_{j}\right| l_{j} \cos \alpha_{j}\right) \\
& =(-2)^{n} \Delta^{2} \cdot \prod_{j=1}^{n}\left|h_{j}\right| l_{j} \cos \alpha_{j}= \pm 2^{n} \Delta^{2} \cdot \prod_{j=1}^{n}\left|h_{j}\right| l_{j} \neq 0 .
\end{aligned}
$$

\subsection{A singular example with small divisors}

Consider now the Hamiltonian $H=H_{0}+\mu H_{1}$, with

$$
\begin{aligned}
& H_{0}(x, y, I)=\langle\omega, I\rangle+\frac{1}{2}\langle\Lambda I, I\rangle+\frac{y^{2}}{2}+\cos x-1, \\
& H_{1}(x, \varphi)=(\cos x-1) f(\varphi), \quad f(\varphi)=\sum_{k \in \mathbb{Z}^{n}} f_{k} e^{i\langle k, \varphi\rangle} .
\end{aligned}
$$

The integrable Hamiltonian $H_{0}$ is uncoupled $(\lambda=0$ in (2)), and consists of a pendulum and $n$ rotors. Note also that the perturbation $H_{1}$ depends only on the angles $x, \varphi$ and that, since $H_{1}=\mathrm{O}_{2}(x)$, the whiskered torus remains fixed (as in the original Arnold's example [Arn64]). However, following [Chi79, p. 358] and [Loc92, p. 117] we will not assume that $f(\varphi)$ is a trigonometric polynomial. On the contrary, we assume an analytic perturbation, with exponentially decreasing coefficients:

$$
\left|f_{k}\right| \leq e^{-|k| \rho} \quad \forall k \in \mathbb{Z}^{n} \backslash\{0\}
$$

(note that the parameter $\rho$ is the width of analyticity of $H_{1}$ in the angles $\varphi$ ). We also consider fast frequencies $\omega=\omega^{*} / \sqrt{\varepsilon}$, because we are interested in a singular situation.

The fact that $H_{1}=\mathrm{O}_{2}(x)$ allows us to compute the Melnikov potential applying the simple formula (37). Using the expression (44) for the homoclinic trajectory of the standard pendulum, we have

$$
L(\varphi)=-\int_{-\infty}^{\infty}\left(\cos x_{0}(t)-1\right) f(\varphi+\omega t) \mathrm{d} t+\text { const }=2 \int_{-\infty}^{\infty} \frac{f(\varphi+\omega t)}{\cosh ^{2} t} \mathrm{~d} t+\text { const. }
$$

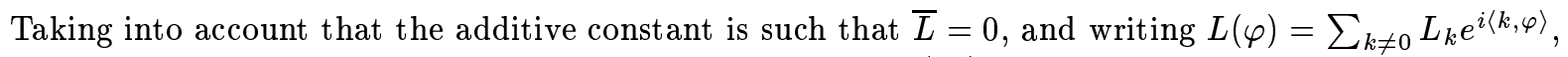
the Fourier coefficients $L_{k}$ can be computed explicitly using (46):

$$
L_{k}=2 f_{k} \int_{-\infty}^{\infty} \frac{e^{i\langle k, \omega\rangle t}}{\cosh ^{2} t} \mathrm{~d} t=\frac{2 \pi\langle k, \omega\rangle f_{k}}{\sinh \left(\frac{\pi}{2}\langle k, \omega\rangle\right)}
$$

(note that the mean value $\bar{f}=f_{0}$ does not influence the Melnikov potential). 


\subsubsection{Upper bounds for the Melnikov potential}

Let us obtain an upper bound of the Melnikov potential $L$ in the case of fast frequencies, showing that its size depends strongly on the small divisors properties of the frequencies. We assume that the vector $\omega^{*}$ is Diophantine, and introduce $\gamma=\gamma^{*} / \sqrt{\varepsilon}$ in (10), for some $\tau \geq n-1$. We have

$$
\left|L_{k}\right| \leq \frac{2 \pi}{\sqrt{\varepsilon}}\left|\left\langle k, \omega^{*}\right\rangle\right| \cdot \frac{e^{-|k| \rho}}{\sinh \left(\frac{\pi}{2 \sqrt{\varepsilon}}\left|\left\langle k, \omega^{*}\right\rangle\right|\right)} .
$$

Now, we use that $\sinh x \geq e^{x} / 3$ for $x \geq 1$, and $\operatorname{simply}$ apply $\sinh x \geq x$ for $0<x<1$. Note that

$$
\left|\left\langle k, \omega^{*}\right\rangle\right| \geq \frac{2 \sqrt{\varepsilon}}{\pi} \quad \text { for } 0<|k| \leq K_{0}=K_{0}(\varepsilon):=\left(\frac{\pi \gamma^{*}}{2 \sqrt{\varepsilon}}\right)^{1 / \tau} .
$$

We obtain the following bounds for the Fourier coefficients:

$$
\begin{aligned}
& \left|L_{k}\right| \leq \frac{6 \pi}{\sqrt{\varepsilon}}\left|\left\langle k, \omega^{*}\right\rangle\right| \exp \left(-|k| \rho-\frac{\pi \gamma^{*}}{2|k|^{\tau} \sqrt{\varepsilon}}\right), \quad 0<|k| \leq K_{0}, \\
& \left|L_{k}\right| \leq 4 e^{-|k| \rho}, \quad|k|>K_{0} .
\end{aligned}
$$

In order to bound the coefficients (48), we use the following property: a function like $g(u)=a u+$ $b u^{-\tau}$, with $a, b>0$, has a minimum at $u^{*}=(\tau b / a)^{1 /(\tau+1)}$, and one has $g\left(u^{*}\right)=(1+1 / \tau) a u^{*}=$ $(1+1 / \tau)\left(\tau a^{\tau} b\right)^{1 /(\tau+1)}$. This implies that the worst bound in (48), for a given $\varepsilon>0$, is given for the indexes $k$ such that

$$
\left|\left\langle k, \omega^{*}\right\rangle\right| \sim \frac{\gamma^{*}}{|k|^{\tau}}, \quad|k| \sim K=K(\varepsilon):=\left(\frac{\pi \tau \gamma^{*}}{2 \rho \sqrt{\varepsilon}}\right)^{1 /(\tau+1)}
$$

and we get

$$
\left|L_{k}\right| \leq \frac{6 \pi}{\sqrt{\varepsilon}}\left|\left\langle k, \omega^{*}\right\rangle\right| e^{-(1+1 / \tau) \rho K}=\frac{6 \pi}{\sqrt{\varepsilon}}\left|\left\langle k, \omega^{*}\right\rangle\right| \exp \left(-C \varepsilon^{-1 /(2 \tau+2)}\right),
$$

where we define

$$
C=\left(1+\frac{1}{\tau}\right)\left(\frac{\pi \gamma^{*} \tau \rho^{\tau}}{2}\right)^{1 /(\tau+1)}
$$

The contribution of the coefficients (49) can be bounded as $\mathrm{O}\left(e^{-K_{0} \rho}\right)$ and, comparing the exponents of $\varepsilon$ in $K_{0}$ and $K$, one sees that this contribution is smaller than (50). Thus, for $\varepsilon>0$ small enough we get an upper bound for the Melnikov potential, exponentially small in $\varepsilon$ :

$$
|L(\varphi)| \leq \frac{\text { const }}{\varepsilon^{1 /(2 \tau+2)}} \exp \left(-C \varepsilon^{-1 /(2 \tau+2)}\right), \quad \varphi \in \mathbb{T}^{n} .
$$

Proceeding analogously for the Melnikov function $M=\partial_{\varphi} L$, whose Fourier coefficients are $M_{k}=i k L_{k}$, one obtains the bound

$$
|M(\varphi)| \leq \frac{\text { const }}{\varepsilon^{1 /(\tau+1)}} \exp \left(-C \varepsilon^{-1 /(2 \tau+2)}\right), \quad \varphi \in \mathbb{T}^{n} .
$$

It is an important point in these estimates to assume a perturbation with an infinite number of harmonics. As stressed in [Loc92, §V2], one is then forced to take into account the small divisors associated to the frequencies, and this leads to the exponent $1 /(2 \tau+2)$ inside the exponential. Notice that this exponent in the upper bound is reminiscent of the Nekhoroshev-like estimates. Instead, if one assumes a finite number of harmonics (like in the Arnold's example [Arn64]), then one obtains the exponent $1 / 2$, but this case is highly nongeneric. 


\subsubsection{Lower bounds for the Melnikov potential in the golden mean case}

A more precise description of the asymptotic behavior of $L$ and $M$ requires a very careful analysis of the small divisors associated to the frequency vector $\omega^{*}$. For the case of 2 frequencies: $\omega^{*}=\left(\omega_{1}^{*}, \omega_{2}^{*}\right)$ (i.e. for $n+1=3$ degrees of freedom), this analysis can be carried out applying the theory of continued fractions to the frequency ratio $\omega_{2}^{*} / \omega_{1}^{*}$. The simplest case is that of the golden mean:

$$
\omega^{*}=(1, \Omega), \quad \Omega=\frac{\sqrt{5}+1}{2},
$$

which was first considered in [Sim94], and later on in [DGJS97], where lower bounds for the Melnikov function and for the splitting were obtained. We point out that such bounds can easily be generalized if the golden mean $\Omega$ in (51) is replaced by another quadratic number $\omega_{2}^{*} / \omega_{1}^{*}$ (see also [RW98] for other different kinds of numbers).

On the other hand, a key point in dealing with the singular case is to assume that, in the perturbation, at least the harmonics $f_{k}$ corresponding to the small divisors associated to $\omega^{*}$ are nonvanishing, because the dominant harmonic is found among these ones. Under this assumption, one can obtain the largest lower bounds (with exponent $1 /(2 \tau+2)$ ) in the Melnikov approximation, in order to ensure that this approximation dominates the $\mathrm{O}\left(\mu^{2}\right)$-remainder, as carried out in [DGJS97].

Next, we shall get a lower bound for the Melnikov potential in the case (51), ensuring also that it has nondegenerate critical points. For the perturbation, in view of the discussion above, we assume that

$$
\left|f_{k}\right|=e^{-|k| \rho} \quad \forall k \in \mathbb{Z}^{2} \backslash\{0\} .
$$

For instance, the function

$$
f(\varphi)=\frac{\sin \varphi_{1}}{\cosh \rho-\cos \varphi_{1}} \cdot \frac{\sinh \rho}{\cosh \rho-\cos \varphi_{2}}
$$

satisfies this requirement. Note that a non-even function $f(\varphi)$ is allowed, so we are not assuming that the perturbation $H_{1}(x, \varphi)$ is reversible (unlike [Gal94, RW98, GGM99]).

It is well-known that the small divisors associated to the golden mean $\Omega$ are directly related to the Fibonacci numbers:

$$
F_{0}=F_{1}=1, \quad F_{n}=F_{n-1}+F_{n-2}, \quad n \geq 2 .
$$

We define also

$$
C_{F}=\frac{1}{\Omega+\Omega^{-1}}=\frac{1}{\sqrt{5}}
$$

and recall that

$$
F_{n-1}=C_{F}\left(\Omega^{n}-(-1)^{n} \Omega^{-n}\right), \quad n \geq 1 .
$$

The best rational approximations of $\Omega$ are given by the convergents $F_{n} / F_{n-1}$. In other words, the indexes $k^{(n)}=\left(F_{n},-F_{n-1}\right)$ (and also $\left(-F_{n}, F_{n-1}\right)$ ) are the ones that give the dominant behavior among the small divisors $\left\langle k, \omega^{*}\right\rangle$. More precisely, one has:

$$
\left\langle k^{(n)}, \omega^{*}\right\rangle=F_{n}-F_{n-1} \Omega=\frac{(-1)^{n}}{\Omega^{n}}=\frac{(-1)^{n} C_{F}}{F_{n-1}}+\mathrm{O}\left(\frac{1}{F_{n-1}^{3}}\right), \quad n \geq 1,
$$

and also the following inequality (see [DGJS97, §6]): for any $k=\left(k_{1},-k_{2}\right)$ such that $k_{2}>0$ is not a Fibonacci number,

$$
\left|\left\langle k, \omega^{*}\right\rangle\right|=\left|k_{1}-k_{2} \Omega\right|>\frac{\Omega C_{F}}{k_{2}} .
$$

Note that the frequency vector $\omega^{*}$ satisfies the Diophantine condition (10) with $\tau=1$.

We are going to show that the dominant harmonics of the Fourier series of the Melnikov potential $L(\varphi)$ are the ones associated to the Fibonacci indexes $k^{(n)}$. We proceed as in [DGJS97], though the context is somewhat different. Denoting $S_{n}=L_{k^{(n)}}$, from (47) we directly obtain

$$
\left|S_{n}\right|=\frac{2 \pi}{\Omega^{n} \sqrt{\varepsilon}} \cdot \frac{e^{-F_{n+1} \rho}}{\sinh \left(\frac{\pi}{2 \Omega^{n} \sqrt{\varepsilon}}\right)}, \quad n \geq 1 .
$$


The main part of this expression is given by

$$
\left|S_{n}^{0}\right|=\frac{4 \pi}{\Omega^{n} \sqrt{\varepsilon}} e^{-b_{n}^{0}}
$$

where we define

$$
b_{n}^{0}=b_{n}^{0}(\varepsilon)=C_{F} \Omega^{n+2} \rho+\frac{\pi}{2 \Omega^{n} \sqrt{\varepsilon}} .
$$

For a given $\varepsilon>0$, to find the dominant harmonic among the Fibonacci ones, we look for the minimum exponent $b_{n}^{0}, n \geq 1$. We introduce

$$
D_{0}=\sqrt{\frac{\pi}{2 C_{F} \rho}}, \quad C_{0}=\Omega \sqrt{2 \pi C_{F} \rho}
$$

and also

$$
\varepsilon_{n}=\left(\frac{D_{0}}{\Omega^{n+1}}\right)^{4}=\frac{\varepsilon_{0}}{\Omega^{4 n}} .
$$

We then obtain the following alternative expression for the exponents (54):

$$
b_{n}^{0}=\frac{C_{0}}{2 \varepsilon^{1 / 4}}\left(\frac{\varepsilon^{1 / 4} \Omega^{n+1}}{D_{0}}+\frac{D_{0}}{\varepsilon^{1 / 4} \Omega^{n+1}}\right)=\frac{C_{0}}{\varepsilon^{1 / 4}} \cosh \left(\frac{\log \varepsilon-\log \varepsilon_{n}}{4}\right),
$$

and it is clear that the minimum exponent is reached when $\log \varepsilon_{n}$ is closest to $\log \varepsilon$.

To analyze better how the minimum value depends on $\varepsilon$, we consider $\delta=\log \varepsilon$, and $\delta_{n}=\log \varepsilon_{n}=$ $\delta_{0}-4 n \log \Omega$. For a given $\delta$, the minimum among the $\left|\delta-\delta_{n}\right|$ is reached by only one integer $N_{0}=N_{0}(\delta)$, except for the case that $\delta$ is some $\left(\delta_{n}+\delta_{n+1}\right) / 2$, in which the minimum is reached for two integers $N_{0}$, $N_{0}+1$. Anyway, it easy to check that the function

$$
\tilde{c}(\delta)=\min _{n}\left|\delta-\delta_{n}\right|=\left|\delta-\delta_{N_{0}}\right|
$$

is $(4 \log \Omega)$-periodic, continuous and piecewise linear, and for any $\delta$ one has

$$
0 \leq \tilde{c}(\delta) \leq 2 \log \Omega
$$

The extreme values 0 and $2 \log \Omega$ are obtained for $\delta=\delta_{n}$ and $\delta=\left(\delta_{n}+\delta_{n+1}\right) / 2$, respectively. In fact, we can define $\tilde{c}(\delta)$ as the $(4 \log \Omega)$-periodic extension of

$$
\tilde{c}(\delta)=\left|\delta-\delta_{0}\right|, \quad\left|\delta-\delta_{0}\right| \leq 2 \log \Omega .
$$

Now, consider the function $c(\delta)=\cosh (\tilde{c}(\delta) / 4)$, that is, the $(4 \log \Omega)$-periodic extension of

$$
c(\delta)=C_{0} \cosh \left(\frac{\delta-\delta_{0}}{4}\right), \quad\left|\delta-\delta_{0}\right| \leq 2 \log \Omega .
$$

This function satisfies

$$
C_{0} \leq c(\delta) \leq \frac{\Omega^{3 / 2} C_{0}}{2}=(1.029085 \ldots) C_{0} .
$$

In terms of $\varepsilon$, we see that the minimum exponent $b_{n}^{0}$ is given by

$$
b_{N_{0}}^{0}=\frac{c(\log \varepsilon)}{\varepsilon^{1 / 4}}
$$

and this implies that the coefficient $S_{N_{0}}^{0}$ is the dominant one among the $S_{n}^{0}$. Note that one has $N_{0} \geq 1$ provided $\delta \leq \delta_{0}-2 \log \Omega$, i.e. for $\varepsilon \leq \varepsilon_{0} / \Omega^{2}$.

Now, to see that the "whole" coefficient $S_{N_{0}}$ is also dominant among the $S_{n}$, we write these coefficients in the form

$$
\left|S_{n}\right|=\frac{4 \pi}{\Omega^{n} \sqrt{\varepsilon}} e^{-b_{n}}
$$


The new exponents $b_{n}=b_{n}(\varepsilon)$ are related to the previous ones through

$$
b_{n}=b_{n}^{0}-\frac{(-1)^{n} C_{F} \rho}{\Omega^{n+2}}+\log \left(1-\exp \left\{-\frac{\pi}{\Omega^{n} \sqrt{\varepsilon}}\right\}\right),
$$

and it is not hard to see that the new terms in this sum leave unchanged the fact that the minimum exponent is given by $n=N_{0}$. We point out that the dominant coefficient $S_{N_{0}}$ is unique except for the case that $\log \varepsilon$ is close to some $\left(\delta_{n}+\delta_{n+1}\right) / 2$, i.e. for $\varepsilon$ close to some

$$
\varepsilon_{n}^{\prime}=\frac{\varepsilon_{n}}{\Omega^{2}}=\frac{\varepsilon_{0}}{\Omega^{4 n+2}},
$$

where there are two dominant coefficients $S_{N_{0}}, S_{N_{0}+1}$.

The non-Fibonacci coefficients $L_{k}$, for $k \neq k^{(n)}$, do not dominate, since by (52) they can be bounded similarly, but with $\Omega C_{F}$ instead of $C_{F}$. The exponent analogous to the $b_{n}^{0}$ would now be, for the non-Fibonacci indexes $k$,

$$
|k| \rho+\frac{\pi}{2 \sqrt{\varepsilon}}\left|\left\langle k, \omega^{*}\right\rangle\right| \geq \frac{\Omega^{1 / 2} C_{0}}{\varepsilon^{1 / 4}},
$$

which is bigger than the exponent obtained in (56), since $\Omega^{1 / 2}>\Omega^{3 / 2} / 2$. In this way, the maximum value of the Melnikov potential $|L(\varphi)|, \varphi \in \mathbb{T}^{2}$, can be approximated by its dominant Fibonacci harmonic, given by

$$
N_{0}(\varepsilon) \sim \frac{\log \left(\varepsilon_{0} / \varepsilon\right)}{4 \log \Omega}
$$

We obtain, for $\max _{\varphi \in \mathbb{T}^{2}}|L(\varphi)|$, an upper bound and a lower bound, both of the type

$$
\frac{\text { const }}{\varepsilon^{1 / 4}} \exp \left(-\frac{c(\log \varepsilon)}{\varepsilon^{1 / 4}}\right)
$$

\subsubsection{Lower bounds for the determinant at the critical points of the Melnikov potential in the golden mean case}

We now want to show that the Melnikov potential $L(\varphi)$ has nondegenerate critical points, as a first step towards the existence of transverse homoclinic intersections in the singular case. Like in section 3.2, we need to consider at least 2 harmonics in order to find these nondegenerate critical points (recall that here $n=2$ ). Assume $\varepsilon>0$ fixed, not coinciding with any of the $\varepsilon_{n}$. In the discussion above, we can consider also the integer $N_{1}(\varepsilon)$ reaching the "second" minimum among the $b_{n}^{0}$; it is clear that $\left|N_{1}-N_{0}\right|=1$. Calling $N=N(\varepsilon)=\min \left(N_{0}, N_{1}\right)$, it turns out that $\varepsilon_{N+1}<\varepsilon<\varepsilon_{N}$, and the Fibonacci coefficients with indexes $N$ and $N+1$ give the 2 dominant harmonics in the Fourier expansion of the Melnikov potential (note that this discussion is not valid if $\varepsilon$ is one of the $\varepsilon_{n}$, because then the "second" minimum among the $b_{n}^{0}$ and the "third" one reach the same value, and we cannot consider 2 dominant harmonics).

As in section 3.2, it will be suitable to use the trigonometric form of the Fourier expansions. For the function $f(\varphi)$ that gives the perturbation, we write $f_{k} e^{i\langle k, \varphi\rangle}+f_{-k} e^{-i\langle k, \varphi\rangle}=2\left|f_{k}\right| \cos \left(\langle k, \varphi\rangle+\eta_{k}\right)$; note that if $f(\varphi)$ is even (the reversible case), then $\eta_{k}=0$ or $\eta_{k}=\pi$ for any $k$. The main part of the Melnikov potential $L(\varphi)$ is given by its 2 dominant Fibonacci harmonics, with indexes $N, N+1$. This main part can be written as

$$
L^{(N)}(\varphi)=2\left|S_{N}\right| \cos \left(\left\langle k^{(N)}, \varphi\right\rangle+\sigma_{N}\right)+2\left|S_{N+1}\right| \cos \left(\left\langle k^{(N+1)}, \varphi\right\rangle+\sigma_{N+1}\right),
$$

where we denote $\sigma_{N}=\eta_{k^{(N)}}$. We can apply to this trigonometric polynomial the results of the end of section 3.2. So we consider the determinant

$$
\Delta_{N}=k_{1}^{(N)} k_{2}^{(N+1)}-k_{2}^{(N)} k_{1}^{(N+1)}=F_{N-1} F_{N+1}-F_{N}^{2}=(-1)^{N+1},
$$

and hence $L^{(N)}(\varphi)$ has exactly 4 critical points $\varphi^{*}$. All of them are nondegenerate:

$$
\left|\operatorname{det} \partial_{\varphi}^{2} L^{(N)}\left(\varphi^{*}\right)\right|=4\left|S_{N} S_{N+1}\right| \neq 0 .
$$


Again, we can use (53) to obtain the main part of this determinant:

$$
4\left|S_{N}^{0} S_{N+1}^{0}\right|=\frac{64 \pi^{2}}{\Omega^{2 N+1} \varepsilon} e^{-\left(b_{N}^{0}+b_{N+1}^{0}\right)} .
$$

Using (54) and proceeding as before, we obtain expressions for the new exponent, analogous to (55) and (56):

$$
b_{N}^{0}+b_{N+1}^{0}=C_{F} \Omega^{N+4} \rho+\frac{\pi}{2 \Omega^{N-1} \sqrt{\varepsilon}}=\frac{C_{1}}{\varepsilon^{1 / 4}} \cosh \left(\frac{\log \varepsilon-\log \varepsilon_{N}^{\prime}}{4}\right)=\frac{c_{1}(\log \varepsilon)}{\varepsilon^{1 / 4}} .
$$

Here, we define

$$
C_{1}=\Omega^{3 / 2} C_{0}=\Omega^{5 / 2} \sqrt{2 \pi C_{F} \rho}
$$

and $c_{1}(\delta)$ is a $(4 \log \Omega)$-periodic function, defined from

$$
c_{1}(\delta)=C_{1} \cosh \left(\frac{\delta-\delta_{0}^{\prime}}{4}\right), \quad\left|\delta-\delta_{0}^{\prime}\right| \leq 2 \log \Omega
$$

with $\delta_{0}^{\prime}=\log \varepsilon_{0}^{\prime}$. It is easy to find the extreme values of this function:

$$
C_{1} \leq c_{1}(\delta) \leq \frac{\Omega^{3 / 2} C_{1}}{2}
$$

Now, we use that the trigonometric polynomial $L^{(N)}(\varphi)$ gives the main contribution to $L(\varphi)$. We obtain, at the 4 critical points, an upper bound and a lower bound for the determinant of $\partial_{\varphi}^{2} L$, both of the type

$$
\frac{\text { const }}{\varepsilon^{1 / 2}} \exp \left(-\frac{c_{1}(\log \varepsilon)}{\varepsilon^{1 / 4}}\right)
$$

We recall that this determinant is a measure for the transversality of the splitting. Finally, it is a direct consequence of proposition 5 that, for $\mu=o\left(\exp \left\{-c_{1}(\log \varepsilon) \varepsilon^{-1 / 4}\right\}\right)$, there exist 4 transverse homoclinic intersections, as predicted by the Melnikov potential. However, we recall that this is actually a regular situation, and a justification for the singular case $\mu=\varepsilon^{p}$, for some $p>0$, does not follow directly from proposition 5 .

\subsection{Melnikov integrals for a heteroclinic case}

Now, we consider the Hamiltonian (1-2) without assuming that the perturbation $H_{1}$ is periodic with respect to the variable $x$. In this case, the unperturbed tori at $x=0$ and at $x=2 \pi$ must be considered as different: we denote them $\mathcal{T}_{0}^{(m)}, m=0,2 \pi$. For the unperturbed Hamiltonian $H_{0}$, we have a heteroclinic connection: the unstable whisker of $\mathcal{T}_{0}^{(0)}$ and the stable whisker of $\mathcal{T}_{0}^{(2 \pi)}$ coincide. Applying the hyperbolic KAM theorem in a neighborhood of each torus, under the usual assumptions, we get perturbed tori $\mathcal{T}^{(m)}$ as well as their local whiskers. Extending these local whiskers to global ones, our aim is to measure the splitting between the global unstable whisker $\mathcal{W}^{(0),-}$ of $\mathcal{T}^{(0)}$ and the global stable whisker $\mathcal{W}^{(2 \pi),+}$ of $\mathcal{T}^{(2 \pi)}$.

Proceeding analogously to sections 2.2 and 2.3, we can define parameterizations $z^{(m), *}(\varphi)$ and $z^{(m), \pm}(s, \varphi)$ for the tori $\mathcal{T}^{(m)}$ and the whiskers $\mathcal{W}^{(m), \pm}$, respectively.

It has to be pointed out that the perturbed tori $\mathcal{T}^{(0)}$ and $\mathcal{T}^{(2 \pi)}$ do not necessarily lie in the same energy level (indeed, we cannot expect the additive constant in the normal form (16) to be the same for the two tori, because it comes from applying the KAM theorem independently to each one). If their energy is not the same, the whiskers $\mathcal{W}^{(0),-}$ and $\mathcal{W}^{(2 \pi),+}$ do not intersect. However, in the particular case in which they lie in the same energy level, we can measure the splitting distance by considering the difference between the parameterizations of the global whiskers, along the $I$-direction: $I^{(0),-}-I^{(2 \pi),+}$, and we want to obtain the first order approximation in $\mu$. Writing

$$
\begin{aligned}
I^{(0),-}(s, \varphi)-I^{(2 \pi),+}(s, \varphi)= & \left(I^{(0),-}(s, \varphi)-I^{(0), *}(\varphi-\pi \lambda)\right)-\left(I^{(2 \pi),+}(s, \varphi)-I^{(2 \pi), *}(\varphi+\pi \lambda)\right) \\
& +\left(I^{(0), *}(\varphi-\pi \lambda)-I^{(2 \pi), *}(\varphi+\pi \lambda)\right)
\end{aligned}
$$


we then proceed like in the proof of proposition 5. The only difference lies in the fact that the shift of the tori $\mathcal{T}^{(m)}$, with respect to the unperturbed ones, is not necessarily the same for the two tori. More precisely, lemma 2 provides different approximations for the two perturbed tori:

$$
\begin{aligned}
& I^{(0), *}(\varphi-\pi \lambda)=\mu\left(\zeta^{(0)}-\partial_{\varphi} \chi^{(0)}(\varphi-\pi \lambda)\right)+\mathrm{O}\left(\mu^{2}\right), \\
& I^{(2 \pi), *}(\varphi+\pi \lambda)=\mu\left(\zeta^{(2 \pi)}-\partial_{\varphi} \chi^{(2 \pi)}(\varphi+\pi \lambda)\right)+\mathrm{O}\left(\mu^{2}\right),
\end{aligned}
$$

where each $\chi^{(m)}(\varphi)$ solves the small divisors equation

$$
\left\langle\omega, \partial_{\varphi} \chi^{(m)}\right\rangle+\overline{H_{1}}(m, 0,0)=H_{1}(m, 0, \cdot, 0), \quad m=0,2 \pi,
$$

and the mean value of the shift is given at first order by

$$
\zeta^{(m)}=-\widehat{\Lambda}^{-1}\left(\overline{\partial_{I} H_{1}-\lambda \partial_{y} H_{1}}\right)(m, 0,0), \quad m=0,2 \pi .
$$

Taking into account these facts, we get the following first order approximation that generalizes (38):

$$
I^{(0),-}(s, \varphi)-I^{(2 \pi),+}(s, \varphi)=\mu\left(\zeta^{(0)}-\zeta^{(2 \pi)}+M(\varphi-\omega s)\right)+\mathrm{O}\left(\mu^{2}\right) .
$$

Here, the Melnikov function is again a gradient: $M(\varphi)=\partial_{\varphi} L(\varphi)$, and the Melnikov potential can be defined as the following modified version for the formula (34), with correcting terms associated to $\chi^{(0)}$ and $\chi^{(2 \pi)}$ :

$$
L(\varphi)=\lim _{T \rightarrow \infty}\left[-\int_{-T}^{T}\left(H_{1}-\overline{H_{1}}\right)\left(z_{0}(t, \varphi+\omega t)\right) \mathrm{d} t+\chi^{(0)}(\varphi+\pi \lambda+\omega T)-\chi^{(2 \pi)}(\varphi-\pi \lambda-\omega T)\right] .
$$

We are interested in expressing the Melnikov potential by means of an absolutely convergent integral that includes the correcting terms, like in (31). The equivalence between formulas (31) and (34) shown in section 3.1 cannot be generalized here because, in general, $\chi^{(0)} \neq \chi^{(2 \pi)}$. Nevertheless, we can consider a function $\tilde{\chi}$ that "interpolates" $\chi^{(0)}$ and $\chi^{(2 \pi)}$. A way to construct such an interpolation is to choose $\tilde{\chi}(x, y, \varphi, I)$ solving the small divisors equation

$$
\left\langle\omega, \partial_{\varphi} \tilde{\chi}\right\rangle+\overline{H_{1}}=H_{1} .
$$

One obtains a formula for $L(\varphi)$ analogous to (31), replacing $\chi$ by $\tilde{\chi}$. Besides, it is easy to check that $H_{1}-\overline{H_{1}}-\left\{\tilde{\chi}, H_{0}\right\}$ coincides with $-\{\tilde{\chi}, \widehat{P}\}$ on $I=0$ (recall that $\widehat{P}$ was defined in (6)). We then obtain the following simple expression, which does not depend on the choice of $\tilde{\chi}$ :

$$
L(\varphi)=\int_{-\infty}^{\infty}\{\tilde{\chi}, \widehat{P}\}\left(z_{0}(t, \varphi+\omega t)\right) \mathrm{d} t .
$$

We point out that, even if both whiskers lie in the same energy level, the constants $\zeta^{(0)}$ and $\zeta^{(2 \pi)}$ appearing in (57), associated to the shift of the tori, make it more involved to find transverse heteroclinic intersections in the regular case, because they are not given by the nondegenerate critical points of $L$, when $\zeta^{(0)} \neq \zeta^{(2 \pi)}$. Also, to detect heteroclinic intersections from the Melnikov function $M$, it is necessary that the maximum of $|M|$ is greater than the difference $\left|\zeta^{(2 \pi)}-\zeta^{(0)}\right|$. The situation becomes even more disappointing in the singular case, because $M$ will be typically exponentially small in $\varepsilon$; then to find heteroclinic intersections one needs that the mean shift of the two tori is the same to all orders (for instance, assuming that the two tori remain unchanged under the perturbation).

As an illustration for the formula (59), we consider the example provided in [HM82] (see also [Wig88, $\S 4.2 \mathrm{~d}])$. This is a Hamiltonian of the type (1-2), with $n+1=3$ degrees of freedom, $V(x)=\cos x-1$, $\lambda=0$, and the following perturbation:

$$
H_{1}=\frac{1}{2} \sum_{j=1}^{2}\left(\sqrt{2 I_{j}} \sin \varphi_{j}-x\right)^{2}
$$


In [HM82], the measure of the splitting in this example is computed directly using (derivatives of) integrals like (37), which are conditionally convergent in this case. Note that the perturbation $H_{1}$ is not periodic in $x$; so this example is more properly dealt with the formula (59), suitable for the heteroclinic case. From (58), we can take

$$
\tilde{\chi}=\sum_{j=1}^{2}\left(-\frac{1}{4 \omega_{j}} I_{j} \sin 2 \varphi_{j}+\frac{\sqrt{2 I_{j}}}{\omega_{j}} x \cos \varphi_{j}\right) .
$$

On a fixed plane $I=I^{0}$ (instead of $I=0$ ), we apply (59) but writing $z_{0}(s, \varphi)=\left(x_{0}(s), y_{0}(s), \varphi, I^{0}\right)$, and obtain the following Melnikov potential:

$$
L(\varphi)=\sum_{j=1}^{2} \frac{\sqrt{2 I_{j}^{0}}}{\omega_{j}} \int_{-\infty}^{\infty} y_{0}(t) \cos \left(\varphi_{j}+\omega_{j} t\right) \mathrm{d} t=\sum_{j=1}^{2} \frac{2 \pi \sqrt{2 I_{j}^{0}}}{\omega_{j} \cosh \left(\pi \omega_{j} / 2\right)} \cos \varphi_{j}
$$

where we have used (44) and (46) in computing the integral. The subsequent expression for the Melnikov function is in agreement with the results given in [HM82].

It is clear that $L(\varphi)$ has four nondegenerate critical points at $(0,0),(0, \pi),(\pi, 0),(\pi, \pi)$. Besides, in this example we have $\overline{\partial_{I} H_{1}}\left(0,0, I^{0}\right)=\overline{\partial_{I} H_{1}}\left(2 \pi, 0, I^{0}\right)=\left(\frac{1}{2}, \frac{1}{2}\right)$, and hence the shifts associated to the two tori coincide at first order: $\zeta^{(0)}=\zeta^{(2 \pi)}$. Nevertheless, the critical points obtained do not give rise to transverse heteroclinic orbits, because the tori lie in different energy levels. Indeed, a simple computation shows that the first order term of the difference of energies is $\left\langle\omega, \zeta^{(0)}-\zeta^{(2 \pi)}\right\rangle+$ $\bar{H}_{1}\left(0,0, I^{0}\right)-\bar{H}_{1}\left(2 \pi, 0, I^{0}\right)=-2 \pi^{2}$.

As a second illustration, we show that the study of any Hamiltonian of the type (1-2), homoclinic (periodic in $x$ ) and coupled $(\lambda \neq 0)$, can be reduced to a heteroclinic and uncoupled Hamiltonian. Indeed, considering the (exact) symplectic change of variables

$$
Q(x, y, \varphi, I)=(x, y-\langle\lambda, I\rangle, \varphi+\lambda x, I)
$$

we obtain the Hamiltonian $\check{H}=H \circ Q=\check{H}_{0}+\mu \check{H}_{1}$, with

$$
\begin{aligned}
& \check{H}_{0}=\langle\omega, I\rangle+\frac{1}{2}\langle\widehat{\Lambda} I, I\rangle+P(x, y), \\
& \check{H}_{1}=H_{1}(x, y-\langle\lambda, I\rangle, \varphi+\lambda x, I),
\end{aligned}
$$

and the new perturbation is not periodic in $x$ (unless $\lambda$ is integer). Concerning the parameterization for the unperturbed whisker, note that $Q\left(\check{z}_{0}(s, \varphi)\right)=Q\left(x_{0}(s), y_{0}(s), \varphi, 0\right)=z_{0}(s, \varphi+\pi \lambda)$ (so the change $Q$ removes the phase drift term (11)). The transformed equation (58) is now solved by the function $\check{\chi}=\tilde{\chi} \circ Q$. Then we see from (59) that the Melnikov potential remains essentially the same:

$$
\check{L}(\varphi)=\int_{-\infty}^{\infty}\{\check{\chi}, P\}\left(x_{0}(t), y_{0}(t), \varphi+\omega t, 0\right) \mathrm{d} t=\int_{-\infty}^{\infty}\{\tilde{\chi}, \widehat{P}\} \circ Q\left(x_{0}(t), y_{0}(t), \varphi+\omega t, 0\right) \mathrm{d} t=L(\varphi+\pi \lambda) .
$$

In this case, it is clear that the two tori lie in the same energy level, and we can also check that $\zeta^{(0)}=\zeta^{(2 \pi)}$ for the transformed heteroclinic Hamiltonian.

\section{Flow-box variables}

Returning to the homoclinic case, our aim is now to provide a clearer formulation for the problem of measuring the splitting. To reach this, it is convenient to introduce new symplectic variables, called flow-box variables, in which the Hamiltonian equations are very simple. In the neighborhood where we define these variables, we can make the local stable whisker become a coordinate plane. Thanks to the use of the Kolmogorov's approach to the hyperbolic KAM theory, this neighborhood also contains a piece of the global unstable whisker, which can be seen as a graphic over the local stable one. The flow-box variables $(S, E, \psi, J)$ will be defined in such a way that the stable whisker is given by the equations 
$E=0, J=a$, and the variables $S, \psi$ coincide with the parameters $s, \varphi$ on this whisker. The variables $J$ are inherited from the initial variables $I$, and the variable $E$ (conjugate to $S$ ), introduced in order to have a symplectic change, is related to the energy.

Analogous flow-box variables are also used in [DS97, DGJS97] but, in these papers, the change can be defined explicitly from the expression of the normal form, which is integrable. In our case, the flow-box variables are more involved because, in general, the normal form $\widetilde{H}$ obtained in (16) is not integrable.

Our construction of the flow-box variables is a standard one for a Hamiltonian system (see for instance [AM78, §5.2]), but we have to be careful in order to obtain a global domain in the angle variables. We start from the expression of the normal form $\widetilde{H}$, and consider in these variables an $n$-dimensional $\varphi$-section on the local stable whisker:

$$
\mathcal{S}=\tilde{z}\left(s_{1}, \mathbb{T}^{n}\right) \subset \widetilde{\mathcal{W}}_{\text {loc }}^{+},
$$

with some $s_{1}>s_{0}$. To fix ideas, we can take $s_{1}=2 s_{0}$. The $\varphi$-section $\mathcal{S}$ is contained in the $(2 n+1)$ dimensional $(y, \varphi, I)$-section $\Sigma$ given by $x=x_{0}\left(b s_{1}\right)$ (this is a Poincaré section). To parameterize $\Sigma$ in terms of the energy, we consider (from the implicit function theorem) the real-analytic function $\bar{y}(E, \psi, J)=\bar{y}(E, \psi, J ; \mu)$, with $\bar{y}(0, \psi, 0 ; 0)=y_{0}\left(s_{1}\right)$ for any $\psi \in \mathbb{T}^{n}$, solving the equation

$$
\widetilde{H}\left(x_{0}\left(b s_{1}\right), \bar{y}(E, \psi, J), \psi+\xi_{0}\left(b s_{1}\right), J\right)=E+\langle\omega, J-a\rangle
$$

(first we solve it locally for a given $\psi$, and then using the compacity of $\mathbb{T}^{n}$ we obtain a global solution). So the section $\Sigma$ can be parameterized, for $|\mu|$ small enough, as

$$
\Sigma: \quad \sigma(E, \psi, J)=\left(x_{0}\left(b s_{1}\right), \bar{y}(E, \psi, J), \psi+\xi_{0}\left(b s_{1}\right), J\right), \quad|E|,|J| \leq \hat{r}, \quad \psi \in \mathbb{T}^{n},
$$

with $\hat{r}$ not depending on $\omega, \mu$. For any $\psi \in \mathbb{T}^{n}$ we have

$$
\sigma(0, \psi, a)=\tilde{z}\left(s_{1}, \psi\right)
$$

and therefore $\sigma\left(0, \mathbb{T}^{n}, a\right)=\mathcal{S}$.

Since the Hamiltonian flow associated to $\widetilde{H}$ is transversal to the section $\Sigma$ on the points of $\mathcal{S}$, taking initial conditions on $\Sigma$ we cover a whole neighborhood of $\mathcal{S}$. Denote $\widetilde{\Upsilon}^{t}=\widetilde{\Upsilon}^{t}(\cdot ; \mu)$ the time- $t$ flow of $\widetilde{H}$, with real $t$. Then we introduce the flow-box variables $W=(S, E, \psi, J)$ on a neighborhood of $\widehat{\mathcal{S}}=\left(0,0, \mathbb{T}^{n}, a\right)$, through the transformation $\Psi=\Psi(\cdot ; \mu)$ defined by

$$
(x, y, \varphi, I)=\Psi(S, E, \psi, J)=\widetilde{\Upsilon}^{S}(\sigma(E, \psi-\omega S, J)) .
$$

We have $\Psi(\widehat{\mathcal{S}})=\mathcal{S}$. This transformation is defined, for $|\mu|$ small enough, in a real neighborhood $\mathcal{U}_{\hat{r}}(\widehat{\mathcal{S}})$, whose radius $\hat{r}$ can be relabeled if necessary.

We are going to show next that the transformation $\Psi$ is exact symplectic. Before, notice that our Hamiltonian takes, in the flow-box variables, a very simple form:

$$
\widehat{H}=\widetilde{H} \circ \Psi=E+\langle\omega, J-a\rangle,
$$

and hence the associated Hamiltonian equations are

$$
\dot{S}=1, \quad \dot{E}=0, \quad \dot{\psi}=\omega, \quad \dot{J}=0 .
$$

The next lemma is related to the property that any Hamiltonian flow is exact symplectic (see lemma 8). However, this fact does not apply directly to our transformation $\Psi$ and we have to make a somewhat different proof.

Lemma 6 The transformation $\Psi$ is exact symplectic.

Proof. Note that we can write $\Psi=\Psi_{1} \circ A$, where we define:

$$
\begin{aligned}
& \Psi_{1}(S, E, \psi, J)=\widetilde{\Upsilon}^{S}\left(\sigma_{1}(E, \psi, J)\right), \quad \sigma_{1}(E, \psi, J)=\sigma(E-\langle\omega, J-a\rangle, \psi, J), \\
& A(S, E, \psi, J)=(S, E+\langle\omega, J-a\rangle, \psi-\omega S, J) .
\end{aligned}
$$


One easily sees that the transformation $A$ is exact symplectic, and hence it suffices to establish the same property for $\Psi_{1}$.

Let us see that the transformation $\Psi_{1}$ is symplectic, from its Jacobian matrix. For $S=0$, the matrix is

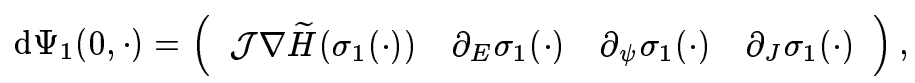

where the dot stands for $(E, \psi, J)$, and $\mathcal{J}$ denotes the standard symplectic matrix. The matrix $\mathrm{d} \Psi_{1}(0, \cdot)$ is symplectic, as verified directly using the expression of $\sigma$ introduced in (61). For $S \neq 0$, we can check that

$$
\mathrm{d} \Psi_{1}(S, \cdot)=\mathrm{d} \widetilde{\Upsilon}^{S}\left(\sigma_{1}(\cdot)\right) \mathrm{d} \Psi_{1}(0, \cdot),
$$

using for the first column that $\mathcal{J} \nabla \widetilde{H} \circ \widetilde{\Upsilon}^{S}=\mathrm{d} \widetilde{\Upsilon}^{S} \mathcal{J} \nabla \widetilde{H}$, and verifying the other columns directly. Thus, the matrix $d \Psi_{1}$ is symplectic at any point, since so is $\mathrm{d} \widetilde{\Upsilon}^{S}$.

In order to check that $\Psi_{1}$ is exact, we try to proceed more or less like in lemma 8. Considering the 1-form $\eta$ introduced in (15), and using that $\partial_{S} \Psi_{1}=\mathcal{J} \nabla \widetilde{H} \circ \Psi_{1}$, we have

$$
\partial_{S}\left(\Psi_{1}^{*} \eta\right)=\mathrm{d}\left[(\widetilde{H}+\eta \cdot \mathcal{J} \nabla \widetilde{H}) \circ \Psi_{1}\right] .
$$

To see that $\Psi_{1}^{*} \eta-\eta=\zeta_{S} \mathrm{~d} S+\zeta_{E} \mathrm{~d} E+\zeta_{\psi} \mathrm{d} \psi+\zeta_{J} \mathrm{~d} J$ has a global primitive function, we cannot proceed exactly as in lemma 8 because the transformation $\Psi_{1}$ does not coincide with the identity on $S=0$. Nevertheless, in finding the primitive function it suffices to control the $\mathrm{d} \psi$-component $\zeta_{\psi}$ of the 1-form. For this component, using that the $J$-component of $\Psi_{1}$ does coincide with $J$ on $S=0$, it is not hard to check that $\zeta_{\psi}(0, \cdot)=0$. Then from $(64)$, we obtain:

$$
\zeta_{\psi}(S, \cdot)=\int_{0}^{S} \partial_{S^{\prime}} \zeta_{\psi}\left(S^{\prime}, \cdot\right) \mathrm{d} S^{\prime}=\partial_{\psi}\left[\int_{0}^{S}(\widetilde{H}+\eta \cdot \mathcal{J} \nabla \widetilde{H}) \circ \Psi_{1}\left(S^{\prime}, \cdot\right) \mathrm{d} S^{\prime}\right],
$$

and we deduce that $\Psi_{1}$ is exact.

Let us denote $\widehat{\mathcal{W}}_{\text {loc }}^{+}=\Psi^{-1}\left(\widetilde{\mathcal{W}}_{\text {loc }}^{+}\right)$the local stable whisker (or more precisely a piece of it) expressed in the flow-box variables. From (62) and the simple form (63) of the Hamiltonian equations in the flow-box variables, we obtain

$$
\Psi(s, 0, \varphi, a)=\tilde{\Upsilon}^{s}(\sigma(0, \varphi-\omega s, a))=\tilde{\Upsilon}^{s}\left(\tilde{z}\left(s_{1}, \varphi-\omega s\right)\right)=\tilde{z}\left(s_{1}+s, \varphi\right) .
$$

Then it is clear that this whisker becomes a coordinate plane, given by $E=0, J=a$, and can be parameterized as follows:

$$
\widehat{\mathcal{W}}_{\mathrm{loc}}^{+}: \quad W_{\mathrm{loc}}^{+}(s, \varphi)=\Psi^{-1}\left(\tilde{z}\left(s_{1}+s, \varphi\right)\right)=(s, 0, \varphi, a), \quad|s| \leq \hat{r}, \quad \varphi \in \mathbb{T}^{n}
$$

(we have replaced $s$ by $s_{1}+s$ for a clearer notation: in this way we have $s=0$ on the selected section $\widehat{\mathcal{S}})$.

Now, we define $\widehat{\mathcal{W}}^{-}=\Psi^{-1} \circ \Phi^{-1}\left(\mathcal{W}^{-}\right)$as an invariant manifold of $\widehat{H}$, which is the equivalent in the flow-box variables for (a piece of) the global unstable whisker. Let us parameterize:

$$
\widehat{\mathcal{W}}^{-}: \quad W^{-}(s, \varphi)=\Psi^{-1} \circ \Phi^{-1}\left(z^{-}(s, \varphi)\right), \quad|s| \leq \hat{r}^{\prime}, \quad \varphi \in \mathbb{T}^{n}
$$

with some $\hat{r}^{\prime}<\hat{r}$. In components, we write

$$
W^{-}(s, \varphi)=\left(S^{-}(s, \varphi), E^{-}(s, \varphi), \psi^{-}(s, \varphi), J^{-}(s, \varphi)\right) .
$$

There is splitting when $J^{-}(s, \varphi) \neq a$ or $E^{-}(s, \varphi) \neq 0$. Nevertheless, since the whisker is contained in the zero energy level, we have $E^{-}+\left\langle\omega, J^{-}-a\right\rangle=0$ and it suffices to control the $J$-component. Note that, if one has $J^{-}\left(s_{0}, \varphi_{0}\right)=a$ for some concrete values $\left(s_{0}, \varphi_{0}\right)$, then the associated homoclinic trajectory is given by $t \mapsto\left(s_{0}+t, 0, \varphi_{0}+\omega t, a\right)$. Thus, the homoclinic trajectories and the splitting have a very simple formulation when expressed in the flow-box variables. 
Using that $z^{-}-z_{\text {loc }}^{+}=\mathrm{O}(\mu)$ (as deduced from lemmas 3 and 4), one sees that the functions $S^{-}-s$, $E^{-}, \psi^{-}-\varphi, J^{-}-a$ are all $\mathrm{O}(\mu)$. Using the simple form (63) of the Hamiltonian equations, one sees also that these functions only depend on $\varphi-\omega s$ (so they are quasiperiodic in $s$ ):

$$
\begin{aligned}
& S^{-}(s, \varphi)-s=S^{-}(0, \varphi-\omega s), \\
& E^{-}(s, \varphi)=E^{-}(0, \varphi-\omega s), \\
& \psi^{-}(s, \varphi)-\varphi=\psi^{-}(0, \varphi-\omega s)-(\varphi-\omega s), \\
& J^{-}(s, \varphi)=J^{-}(0, \varphi-\omega s) .
\end{aligned}
$$

In fact, a stronger result will be obtained in section 5.2, after giving the unstable whisker $\widehat{\mathcal{W}}^{-}$another parameterization.

The next result says that the approximation given in proposition 5, expressed there in the original variables, remains true after changing to the flow-box variables. So the Melnikov function $M$ also provides a first order approximation in $\mu$ for the difference $J^{-}-a$, at least in the regular case.

Proposition 7 For any $|s| \leq \hat{r}^{\prime}$ and $\varphi \in \mathbb{T}^{n}$, one has

$$
J^{-}(s, \varphi)-a=\mu M(\varphi-\omega s)+\mathrm{O}\left(\mu^{2}\right) .
$$

Proof. Consider the projection $\mathcal{I}(S, E, \psi, J)=J$, and denote $\mathcal{I}_{1}=\mathcal{I} \circ \Psi^{-1} \circ \Phi^{-1}-\mathcal{I}$. It is clear from (65) and (66-67) that, for any $s, \varphi$,

$$
\begin{aligned}
& a=\mathcal{I} \circ \Psi^{-1} \circ \Phi^{-1}\left(z_{\mathrm{loc}}^{+}(s, \varphi)\right)=I_{\mathrm{loc}}^{+}(s, \varphi)+\mathcal{I}_{1}\left(z_{\mathrm{loc}}^{+}(s, \varphi)\right), \\
& J^{-}(s, \varphi)=\mathcal{I} \circ \Psi^{-1} \circ \Phi^{-1}\left(z^{-}(s, \varphi)\right)=I^{-}(s, \varphi)+\mathcal{I}_{1}\left(z^{-}(s, \varphi)\right) .
\end{aligned}
$$

We are going to show that the Jacobian matrix $\mathrm{d} \mathcal{I}_{1}$ is $\mathrm{O}(\mu)$. Then applying proposition 5 , and using that $z^{-}-z_{\text {loc }}^{+}=\mathrm{O}(\mu)$, we get from (69-70) the approximation (68).

To see that $\mathrm{d} \mathcal{I}_{1}=\mathrm{O}(\mu)$, the key point is to use that, although the transformation $\Psi$ is not near to the identity, its $J$-component $\mathcal{I} \circ \Psi$ is near to $\mathcal{I}$. Using the notations introduced in the proof of lemma 6 , we have $\mathcal{I} \circ \Psi-\mathcal{I}=\left(\mathcal{I} \circ \Psi_{1}-\mathcal{I}\right) \circ A$. Taking into account that $\partial_{S}\left(\mathcal{I} \circ \Psi_{1}\right)=-\partial_{\varphi} \widetilde{H} \circ \Psi_{1}$, and that $\mathcal{I} \circ \Psi_{1}$ coincides with $\mathcal{I}$ on $S=0$, we can write

$$
\mathcal{I} \circ \Psi_{1}(S, \cdot)-\mathcal{I}=-\int_{0}^{S} \partial_{\varphi} \widetilde{H} \circ \Psi_{1}\left(S^{\prime}, \cdot\right) \mathrm{d} S^{\prime} .
$$

Note that $\partial_{\varphi} \widetilde{H}=\mathrm{O}(\mu)$, as we see from writing $\widetilde{H}=H_{0}+\left(H_{0} \circ \Phi-H_{0}\right)+\mu H_{1} \circ \Phi$ and using (17). Taking derivatives in the integral with respect to $(S, E, \psi, J)$, we obtain $\mathrm{d}(\mathcal{I} \circ \Psi-\mathcal{I})=\mathrm{O}(\mu)$. On the other hand, we know from (17) that $\Phi-\mathrm{id}=\mathrm{O}(\mu)$, and hence $\mathrm{d} \Phi-\mathrm{Id}=\mathrm{O}(\mu)$. Writing

$$
\mathcal{I}_{1}=-(\mathcal{I} \circ \Psi-\mathcal{I}) \circ \Psi^{-1} \circ \Phi^{-1}-(\mathcal{I} \circ \Phi-\mathcal{I}) \circ \Phi^{-1},
$$

we deduce that $\mathrm{d} \mathcal{I}_{1}=\mathrm{O}(\mu)$.

Remarks.

1. This approximation has been obtained by going back to the original variables. A justification for this is that the approximations carried out in the proof of proposition 5 are possible only in these variables, since the improper integrals involved require to consider whole homoclinic trajectories.

2. The fact that the function $J^{-}(s, \varphi)-a$ (a kind of "splitting distance") only depends on $\varphi-\omega s$ is important in the singular case. The key point (see [DGJS97] and also [DS97]) is to use that a function of $\varphi-\omega s$, with $\omega=\omega^{*} / \sqrt{\varepsilon}$, having a polynomial bound on a complex domain, becomes exponentially small in $\varepsilon$ for real values of $s, \varphi$. This property can give rise to exponentially small estimates for the splitting.

3. The use of some normal form variables (provided by the hyperbolic KAM theorem plus the change to flow-box) is crucial in order to see that $J^{-}(s, \varphi)-a$ only depends on $\varphi-\omega s$. This differs from other approaches [Gal94, RW98], where the splitting function is defined in terms of the original variables (see also a remark after proposition 5 ). 


\section{Splitting potential and homoclinic orbits}

\subsection{An exact symplectic map between the global perturbed whiskers}

As mentioned in section 2.1, the special formulation of theorem 1 allows us to carry out a more global control on the perturbed whiskers. We introduce as in [Eli94] the following integrable Hamiltonian, which is nothing but $H_{0}$ with a properly changed Lyapunov exponent and the variables $y, I$ shifted (compare with (2) and (8)):

$$
\begin{aligned}
N(x, y, I ; \mu) & =\langle\omega, I-a\rangle+\frac{b}{2}\langle\Lambda(I-a), I-a\rangle+b P(x, y+\langle\lambda, a\rangle)+b\langle\lambda, I-a\rangle(y+\langle\lambda, a\rangle) \\
& =\langle\omega, I-a\rangle+\frac{b}{2}\langle\widehat{\Lambda}(I-a), I-a\rangle+b \widehat{P}(x, y, I),
\end{aligned}
$$

where the matrix $\widehat{\Lambda}$ is the one defined in (7). Recall that $a$ and $b$ depend on $\mu$, although this is not made explicit. This Hamiltonian is defined globally in the variable $x \in \mathbb{T}$, and has exactly the same hyperbolic torus $\widetilde{\mathcal{T}}$ and local whiskers $\widetilde{\mathcal{W}}_{\text {loc }}^{ \pm}$, and the dynamics on them, as the local normal form $\widetilde{H}$ given in (16). The only difference is that the parameterization $\tilde{z}(s, \varphi)$, introduced for the local whiskers in (18), can now be defined for any $s \in \mathbb{R}$. In this way the local whiskers $\widetilde{\mathcal{W}}_{\text {loc }}^{ \pm}$can be extended to a (unique) global homoclinic whisker of $N$.

Recall the $\varphi$-section $\mathcal{S} \subset \widetilde{\mathcal{W}}_{\text {loc }}^{+}$introduced in (60), and define also $\mathcal{S}^{\prime}=\tilde{z}\left(-s_{1}, \mathbb{T}^{n}\right) \subset \widetilde{\mathcal{W}}_{\text {loc }}^{-}$. These two $\varphi$-sections are obviously related by the time- $T$ Hamiltonian flow of $N$, with $T=2 s_{1}$ :

$$
\Upsilon_{N}^{T}\left(\mathcal{S}^{\prime}\right)=\mathcal{S}
$$

What comes now is very important. Following [Eli94], we can define in a neighborhood of $\Phi(\mathcal{S})$ the symplectic map

$$
\Theta=\Upsilon^{T} \circ \Phi \circ \Upsilon_{N}^{-T} \circ \Phi^{-1}
$$

Notice that the map $\Theta$ takes a piece of $\mathcal{W}_{\text {loc }}^{+}$(the local stable whisker) into $\mathcal{W}^{-}$(the global unstable whisker). Even more, this map gives in its domain the following correspondence between our parameterizations of the whiskers:

$$
\Theta\left(z_{\text {loc }}^{+}(s, \varphi)\right)=z^{-}(s, \varphi),
$$

as we check easily, using as a crucial point that the Hamiltonians $\widetilde{H}$ and $N$ have the same dynamics on the whiskers:

$$
z_{\text {loc }}^{+}(s, \varphi) \stackrel{\Phi^{-1}}{\longmapsto} \tilde{z}(s, \varphi) \stackrel{\Upsilon_{N}^{-T}}{\longmapsto} \tilde{z}(s-T, \varphi-\omega T) \stackrel{\Phi}{\longmapsto} z_{\mathrm{loc}}^{-}(s-T, \varphi-\omega T) \stackrel{\Upsilon^{T}}{\longmapsto} z^{-}(s, \varphi) .
$$

This suggests that we can consider the difference $\Theta-$ id as a suitable measure for the splitting. Note also that, although other values for $s_{1}$ could have been chosen (in fact, any $s_{1}>s_{0}$ ), the correspondence (72) is independent of $s_{1}$. Instead, what would depend strongly on $s_{1}$ is the neighborhood where $\Theta$ can be defined.

The fact that $\Phi$ is exact symplectic implies, using standard properties, that the map $\Theta$ is also exact symplectic. Indeed, one uses that the exactness is preserved under inversions and compositions. Besides, the following lemma states that a Hamiltonian flow is always exact. This is also a standard fact, but we include its proof here because of its close relation to the proof of lemma 6 .

Lemma 8 The flow $\Upsilon^{t}$ associated to any Hamiltonian $H$ is exact symplectic for any $t$.

Proof. For the 1-form $\eta$ introduced in (15), it has to be checked that $\left(\Upsilon^{t}\right)^{*} \eta-\eta$ has globally a scalar primitive function. Writing $X=\mathcal{J} \nabla H$ the Hamiltonian vector field associated to $H$, one has

$$
\frac{\mathrm{d}}{\mathrm{d} t}\left[\left(\Upsilon^{t}\right)^{*} \eta\right]=\left(\Upsilon^{t}\right)^{*}(\mathrm{~d} \eta \cdot X+\mathrm{d}(\eta \cdot X))=\mathrm{d}\left[(H+\eta \cdot X) \circ \Upsilon^{t}\right]
$$

Then

$$
\left(\Upsilon^{t}\right)^{*} \eta-\eta=\int_{0}^{t} \frac{\mathrm{d}}{\mathrm{d} t^{\prime}}\left[\left(\Upsilon^{t^{\prime}}\right)^{*} \eta\right] \mathrm{d} t^{\prime}=\mathrm{d}\left[\int_{0}^{t}(H+\eta \cdot X) \circ \Upsilon^{t^{\prime}} \mathrm{d} t^{\prime}\right]
$$


The next lemma says that $\Theta$ is near to the identity. In fact, it will be more useful to us, in the following sections, to express this result in terms of the flow-box variables. So we define in a neighborhood of $\widehat{\mathcal{S}}=\left(0,0, \mathbb{T}^{n}, a\right)$ the following map, which is also exact symplectic:

$$
\widehat{\Theta}=\Psi^{-1} \circ \Phi^{-1} \circ \Theta \circ \Phi \circ \Psi \text {. }
$$

We use the notation $\mathcal{U}_{r}(\mathcal{A})$ for a real neighborhood of radius $r$ around a set $\mathcal{A}$.

Lemma 9 For $|\mu|$ small enough, one has $\widehat{\Theta}=\mathrm{id}+\mathrm{O}(\mu)$ on some neighborhood $\mathcal{U}_{\hat{r}^{*}}(\widehat{\mathcal{S}})$, whose radius $\hat{r}^{*}$ does not depend on $\omega, \mu$.

Proof. As a previous step, we consider also the map $\Theta$ expressed in the normal form variables provided by theorem 1 :

$$
\widetilde{\Theta}=\Phi^{-1} \circ \Theta \circ \Phi=\Phi^{-1} \circ \Upsilon^{T} \circ \Phi \circ \Upsilon_{N}^{-T},
$$

defined on a neighborhood of $\mathcal{S}$. Note that $\widehat{\Theta}=\Psi^{-1} \circ \widetilde{\Theta} \circ \Psi$. We are going to check that $\widetilde{\Theta}=\mathrm{id}+\mathrm{O}(\mu)$ on some neighborhood $\mathcal{U}_{\tilde{r}^{*}}(\mathcal{S})$, and this implies that $\widehat{\Theta}=\mathrm{id}+\mathrm{O}(\mu)$ on some neighborhood $\mathcal{U}_{\hat{r}^{*}}(\widehat{\mathcal{S}})$.

Using the variational equations for $N$, we can establish like in (29) the following inequalities: for given $z, z^{\prime}$,

$$
\left|\Upsilon_{N}^{t}\left(z^{\prime}\right)-\Upsilon_{N}^{t}(z)\right| \leq e^{K_{1}^{\prime}|t|}\left|z^{\prime}-z\right|, \quad\left|\Upsilon^{t}(z)-\Upsilon_{N}^{t}(z)\right| \leq K_{2}^{\prime}\left(e^{K_{1}^{\prime}|t|}-1\right)|\mu|,
$$

for any $t$ as long as the flows remain in the domain of $H$. The constants $K_{j}^{\prime}$ do not depend on $\omega, \mu$. Here, the factor $K_{2}^{\prime}|\mu|$ comes from estimating $\nabla(H-N)=\nabla\left(H-H_{0}\right)+\nabla\left(H_{0}-N\right)=\mathrm{O}(\mu)$.

We take $\mathcal{U}_{\tilde{r}^{*}}(\mathcal{S}) \subset B_{\nu r}$ with $\tilde{r}^{*}$ to be determined in such a way that $\widetilde{\Theta}$ is defined on this neighborhood. For a given $z \in \mathcal{U}_{\tilde{r}^{*}}(\mathcal{S})$, let $z^{*} \in \mathcal{S}$ such that $\left|z-z^{*}\right| \leq \tilde{r}^{*}$. We have $\Upsilon_{N}^{-T}\left(z^{*}\right) \in \mathcal{S}^{\prime}$ and, from the first inequality of (73), we get

$$
\left|\Upsilon_{N}^{-T}(z)-\Upsilon_{N}^{-T}\left(z^{*}\right)\right| \leq e^{2 K_{1}^{\prime} s_{1}} \tilde{r}^{*}
$$

and therefore $\Upsilon_{N}^{-T}(z)$ belongs to $B_{\nu r}$ provided $\tilde{r}^{*}$ is small enough. Then using (17), we obtain

$$
\left|\Phi \circ \Upsilon_{N}^{-T}(z)-\Upsilon_{N}^{-T}\left(z^{*}\right)\right| \leq C|\mu|+e^{2 K_{1}^{\prime} s_{1}} \tilde{r}^{*} .
$$

Applying the inequalities (73),

$$
\begin{aligned}
& \left|\Upsilon^{T} \circ \Phi \circ \Upsilon_{N}^{-T}(z)-z^{*}\right| \\
& \quad \leq\left|\Upsilon^{T} \circ \Phi \circ \Upsilon_{N}^{-T}(z)-\Upsilon_{N}^{T} \circ \Phi \circ \Upsilon_{N}^{-T}(z)\right|+\left|\Upsilon_{N}^{T} \circ \Phi \circ \Upsilon_{N}^{-T}(z)-\Upsilon_{N}^{T} \circ \Upsilon_{N}^{-T}\left(z^{*}\right)\right| \\
& \quad \leq K_{2}^{\prime}\left(e^{2 K_{1}^{\prime} s_{1}}-1\right)|\mu|+e^{2 K_{1}^{\prime} s_{1}}\left(C|\mu|+e^{2 K_{1}^{\prime} s_{1}} \tilde{r}^{*}\right) \leq C^{\prime}|\mu|+C^{\prime \prime} \tilde{r}^{*} .
\end{aligned}
$$

Then $\Upsilon^{T} \circ \Phi \circ \Upsilon_{N}^{-T}(z) \in \Phi\left(B_{\nu r}\right)$ provided we take $\tilde{r}^{*}$ and $|\mu|$ small enough, and this ensures that $\widetilde{\Theta}(z)$ is defined on $\mathcal{U}_{\tilde{r}^{*}}(\mathcal{S})$.

Finally, to obtain that $\widetilde{\Theta}=\mathrm{id}+\mathrm{O}(\mu)$, we consider the inequality

$$
\begin{aligned}
|\widetilde{\Theta}(z)-z| \leq & \left|\Phi^{-1} \circ \Upsilon^{T} \circ \Phi \circ \Upsilon_{N}^{-T}(z)-\Upsilon^{T} \circ \Phi \circ \Upsilon_{N}^{-T}(z)\right|+\left|\Upsilon^{T} \circ \Phi \circ \Upsilon_{N}^{-T}(z)-\Upsilon_{N}^{T} \circ \Phi \circ \Upsilon_{N}^{-T}(z)\right| \\
& +\left|\Upsilon_{N}^{T} \circ \Phi \circ \Upsilon_{N}^{-T}(z)-\Upsilon_{N}^{T} \circ \Upsilon_{N}^{-T}(z)\right|
\end{aligned}
$$

and, applying (73) and (17), we can bound the three terms of the right hand side.

Notice that the parameterization (66) for the global unstable whisker $\widehat{\mathcal{W}}^{-}$can also be described in terms of the map $\widehat{\Theta}$. Indeed, taking the correspondence (72) into account, we see that

$$
W^{-}(s, \varphi)=\widehat{\Theta}\left(W_{\mathrm{loc}}^{+}(s, \varphi)\right)=\widehat{\Theta}(s, 0, \varphi, a), \quad|s| \leq \hat{r}^{*}, \quad \varphi \in \mathbb{T}^{n} .
$$




\subsection{Splitting potential and splitting function}

The exactness of the symplectic map $\widehat{\Theta}$ and the fact that $\widehat{\Theta}=\mathrm{id}+\mathrm{O}(\mu)$, imply that for $|\mu|$ small enough there exists a generating function $\theta(S, \check{E}, \psi, \breve{J})$, periodic in the angles $\psi \in \mathbb{T}^{n}$, and defined in some neighborhood $\mathcal{U}_{\hat{r}^{* *}}(\widehat{\mathcal{S}})$ with $\hat{r}^{* *}<\hat{r}^{*}$, such that the map $\widehat{\Theta}:(\check{S}, \check{E}, \check{\psi}, \breve{J}) \mapsto(S, E, \psi, J)$ is given by

$$
S=\check{S}-\partial_{\check{E}} \theta, \quad \check{E}=E-\partial_{S} \theta, \quad \psi=\check{\psi}-\partial_{\breve{J}} \theta, \quad \check{J}=J-\partial_{\psi} \theta .
$$

Notice that the function $\theta=\mathrm{O}(\mu)$ does not include the non-periodic (in $\psi$ ) term $S \check{E}+\langle\psi, \breve{J}\rangle$, which generates the identity part. One can find in [Eli94] how the generating function of any near-to-theidentity exact symplectic map is constructed. However, we point out that flow-box variables are not used in [Eli94] and hence our generating function $\theta$ is not the same as the one used there.

Taking into account (66-67) and (74), we can restrict the equations (75) to the whiskers. We obtain, for $|s| \leq \hat{r}^{* *}$ and $\varphi \in \mathbb{T}^{n}$, the equations

$$
\begin{aligned}
& S^{-}(s, \varphi)=s-\partial_{\breve{E}} \theta\left(S^{-}(s, \varphi), 0, \psi^{-}(s, \varphi), a\right), \\
& E^{-}(s, \varphi)=\partial_{S} \theta\left(S^{-}(s, \varphi), 0, \psi^{-}(s, \varphi), a\right), \\
& \psi^{-}(s, \varphi)=\varphi-\partial_{\breve{J}} \theta\left(S^{-}(s, \varphi), 0, \psi^{-}(s, \varphi), a\right), \\
& J^{-}(s, \varphi)=a+\partial_{\psi} \theta\left(S^{-}(s, \varphi), 0, \psi^{-}(s, \varphi), a\right) .
\end{aligned}
$$

According to the remarks at the end of section 4, one can think that the difference $J^{-}(s, \varphi)-a$ should be the most natural election for the "splitting function". However, it would be better to express the splitting distance as the gradient of some scalar function, because then the splitting can be studied in terms of one only function. We cannot deduce from the equation (79) that $J^{-}(s, \varphi)-a$ is a gradient, but this obstruction is easily overcome with a suitable change of parameters.

We follow the approach introduced in [DS97] for the case of a fast periodic perturbation of a 1-degreeof-freedom Hamiltonian. In order to compare the whiskers $\widehat{\mathcal{W}}_{\text {loc }}^{+}$and $\widehat{\mathcal{W}}^{-}$, it is very suitable to take, as new parameters on $\widehat{\mathcal{W}}^{-}$,

$$
S=S^{-}(s, \varphi), \quad \psi=\psi^{-}(s, \varphi) .
$$

This change is $\mathrm{O}(\mu)$-near to the identity, and can be explicitly inverted from the equations (76) and (78):

$$
s=S+\partial_{\breve{E}} \theta(S, 0, \psi, a), \quad \varphi=\psi+\partial_{\breve{J}} \theta(S, 0, \psi, a) .
$$

In terms of $S, \psi$, the unstable whisker $\widehat{\mathcal{W}}^{-}$appears nicely as a graphic over the stable whisker $\widehat{\mathcal{W}}_{\text {loc }}^{+}$, through the parameterization:

$$
\widehat{\mathcal{W}}^{-}: \quad \tilde{W}^{-}(S, \psi)=\left(S, \tilde{E}^{-}(S, \psi), \psi, \tilde{J}^{-}(S, \psi)\right), \quad|S| \leq \hat{r}^{* *}, \quad \psi \in \mathbb{T}^{n} .
$$

Besides, we see from the equations (77) and (79) that

$$
\begin{aligned}
& \tilde{E}^{-}(S, \psi)=\partial_{S} \theta(S, 0, \psi, a), \\
& \tilde{J}^{-}(S, \psi)=a+\partial_{\psi} \theta(S, 0, \psi, a) .
\end{aligned}
$$

In the new parameters, it is natural to introduce the splitting potential as the following function, periodic in $\psi$ :

$$
\mathcal{L}(S, \psi)=\theta(S, 0, \psi, a), \quad|S| \leq \hat{r}^{* *}, \quad \psi \in \mathbb{T}^{n} .
$$

This function also depends on $\mu$, and is determined up to an additive constant. To fix ideas, we can assume that the generating function $\theta$ has been chosen in such a way that, on $S=0$, the function $\mathcal{L}$ has zero mean: $\overline{\mathcal{L}(0, \cdot)}=0$.

As an important remark, the two main ingredients needed for the definition of the splitting potential appear explicitly in the formula (84): the generating function $\theta$ and the shift $a$. First, the generating function $\theta$ has been defined using that the map $\widehat{\Theta}$ is exact symplectic and close to the identity. Second, 
this map comes from the map $\Theta$, which can be defined in (71) thanks to the fact that the shift $a$ of both whiskers is the same, due to that our problem is homoclinic.

The gradient of $\mathcal{L}$ with respect to the angles,

$$
\mathcal{M}(S, \psi)=\partial_{\psi} \mathcal{L}(S, \psi)
$$

will be our (vector) splitting function. According to the next theorem, the function $\mathcal{M}$ gives a measure for the splitting. We stress that the fact that the splitting distance can be put as the gradient of a potential is a reflection of the Lagrangian properties of the whiskers.

Theorem 10 The functions $\mathcal{L}$ and $\mathcal{M}$ only depend on $\psi-\omega S$ :

$$
\mathcal{L}(S, \psi)=\mathcal{L}(0, \psi-\omega S), \quad \mathcal{M}(S, \psi)=\mathcal{M}(0, \psi-\omega S) .
$$

Besides, the splitting is given in terms of the function $\mathcal{M}$ :

$$
\tilde{J}^{-}(S, \psi)-a=\mathcal{M}(S, \psi) .
$$

Proof. According to the definition of $\mathcal{L}$, the equations $(82-83)$ can be rewritten as

$$
\tilde{E}^{-}(S, \psi)=\partial_{S} \mathcal{L}(S, \psi), \quad \tilde{J}^{-}(S, \psi)=a+\partial_{\psi} \mathcal{L}(S, \psi)
$$

Since the whisker $\widehat{\mathcal{W}}^{-}$is contained in the zero energy level, we have $\tilde{E}^{-}+\left\langle\omega, \tilde{J}^{-}-a\right\rangle=0$, and we get the partial differential equation

$$
\partial_{S} \mathcal{L}(S, \psi)+\left\langle\omega, \partial_{\psi} \mathcal{L}(S, \psi)\right\rangle=0
$$

which implies that $\mathcal{L}$ only depends on $\psi-\omega S$. Of course $\mathcal{M}=\partial_{\psi} \mathcal{L}$ has the same property, and the formula (85) for the splitting is the second equation of (86).

As a corollary of theorem 10, we recover the main result of [Eli94]: there exist at least $n+1$ homoclinic orbits (not necessarily transverse), biasymptotic to the invariant torus $\mathcal{T}$. This result, valid for both the regular case and the singular case, comes from the fact that a function on $\mathbb{T}^{n}$ has at least $n+1$ critical points (not necessarily nondegenerate), according to the Lyusternik-Schnirelman theory (see [CH82, §2.12]). Then for a fixed $S$, the function $\mathcal{L}(S, \cdot)$ has at least $n+1$ critical points, which give rise to respective homoclinic intersections between the whiskers $\mathcal{W}^{ \pm}$, and hence to homoclinic orbits, contained in both whiskers. Note also that, in nondegenerate cases, the number of intersections becomes at least $2^{n}$, as one deduces from Morse theory (see the comments at the end of section 3.1).

Finally, using the Poincaré-Melnikov method we can give a first order approximation, useful in the regular case, for the splitting potential $\mathcal{L}$ and the splitting function $\mathcal{M}$. These functions can be approximated, respectively, by the Melnikov potential $L$ and the Melnikov function $M$ at first order in $\mu$.

Theorem 11 For any $|S| \leq \hat{r}^{* *}$ and $\psi \in \mathbb{T}^{n}$, one has

$$
\mathcal{L}(S, \psi)=\mu L(\psi-\omega S)+\mathrm{O}\left(\mu^{2}\right), \quad \mathcal{M}(S, \psi)=\mu M(\psi-\omega S)+\mathrm{O}\left(\mu^{2}\right) .
$$

Proof. We use the first order approximation given in proposition 7, changing from $s, \varphi$ to the parameters $S, \psi$ introduced by means of (80-81). Recall that this change is $\mathrm{O}(\mu)$-near to the identity. Thus, applying theorem 10 we obtain

$$
\begin{aligned}
\mathcal{M}(S, \psi) & =\tilde{J}^{-}(S, \psi)-a=J^{-}\left(S+\partial_{\breve{E}} \theta(S, 0, \psi, a), \psi+\partial_{\breve{J}} \theta(S, 0, \psi, a)\right)-a \\
& =\mu M\left(\psi-\omega S+\partial_{\breve{J}} \theta(S, 0, \psi, a)-\omega \partial_{\breve{E}} \theta(S, 0, \psi, a)\right)+\mathrm{O}\left(\mu^{2}\right)=\mu M(\psi-\omega S)+\mathrm{O}\left(\mu^{2}\right)
\end{aligned}
$$

(here, the constant in the $\mathrm{O}\left(\mu^{2}\right)$-term may depend on $\omega$ ).

Finally, a simple integration of this approximation provides the Melnikov potential $L$ as the first order approximation for the splitting potential $\mathcal{L}$. The involved additive constant does not appear because $\bar{L}=0$ and we made $\overline{\mathcal{L}(0, \cdot)}=0$ in the definition of $\mathcal{L}$. 
Remark. Although this result only gives a first order approximation for $\mathcal{L}$ in the regular case, one can expect that, under some weak hypotheses on the perturbation, the predictions of the splitting given by the Melnikov potential $L$ are also valid in the singular case $\mu=\varepsilon^{p}$, with some $p>0$. For this case, one would require a significant refinement of theorem 11:

$$
\mathcal{L}(S, \psi)=\mu L\left(\psi-S \omega^{*} / \sqrt{\varepsilon}\right)+\mathrm{O}\left(\mu^{2} \varepsilon^{-p}\right),
$$

for $S, \psi$ on a complex strip $|\operatorname{Im} S| \leq \pi / 2-\varepsilon^{1 / 4},|\operatorname{Im} \psi| \leq \rho-\varepsilon^{1 / 4}$. Such a kind of result requires to extend the flow-box variables to a complex domain, and to apply also an extension theorem.

\section{Acknowledgments}

This work has been supported in part by the Catalan grant CIRIT 1998SGR-00041 and the INTAS project 97-10771. Research by A.D. is also supported by the Spanish grant DGICYT PB94-0215. We would like to thank J.-P. Marco, L. Niederman and D. Sauzin for useful discussions and remarks. We also thank the referee, whose remarks have helped us to improve the structure and clearness of this paper.

\section{References}

[AM78] R. Abraham and J.E. Marsden. Foundations of mechanics. Benjamin/Cummings, Reading, Mass., 2nd edition, 1978.

[Arn63] V.I. Arnold. Proof of a theorem of A.N. Kolmogorov on the invariance of quasi-periodic motions under small perturbations of the Hamiltonian. Russian Math. Surveys, 18(5):9-36, 1963.

[Arn64] V.I. Arnold. Instability of dynamical systems with several degrees of freedom. Soviet Math. Dokl., $5(3): 581-585,1964$.

[BGGS84] G. Benettin, L. Galgani, A. Giorgilli, and J.-M. Strelcyn. A proof of Kolmogorov's theorem on invariant tori using canonical transformations defined by the Lie method. Il Nuovo Cimento B, $79(2): 201-223,1984$.

[Bol95] S.V. Bolotin. Homoclinic orbits in invariant tori of Hamiltonian systems. In V.V. Kozlov, editor, Dynamical systems in classical mechanics, volume 168 of Amer. Math. Soc. Transl. Ser. 2, pages 21-90. Amer. Math. Soc., Providence, RI, 1995. (Adv. Math. Sci., 25).

[CG94] L. Chierchia and G. Gallavotti. Drift and diffusion in phase space. Ann. Inst. H. Poincaré Phys. Théor., 60(1):1-144, 1994.

[CH82] S.-N. Chow and J.K. Hale. Methods of bifurcation theory, volume 251 of Grundlehren Math. Wiss. Springer-Verlag, New York, 1982.

[Chi79] B.V. Chirikov. A universal instability of many-dimensional oscillator systems. Phys. Rep., 52(5):263379, 1979.

[DG98] A. Delshams and P. Gutiérrez. Homoclinic orbits to invariant tori in Hamiltonian systems. In C. Jones, S. Wiggins, A. Khibnik, F. Dumortier, and D. Terman, editors, Multiple-Time-Scale Dynamical Systems (to appear), IMA Volumes in Mathematics and Its Applications. Held in Minneapolis, 27-31 October, 1997. Springer-Verlag, New York, 1998.

[DGJS97] A. Delshams, V.G. Gelfreich, À. Jorba, and T.M. Seara. Exponentially small splitting of separatrices under fast quasiperiodic forcing. Comm. Math. Phys., 189:35-71, 1997.

[DR97] A. Delshams and R. Ramírez-Ros. Melnikov potential for exact symplectic maps. Comm. Math. Phys., 190:213-245, 1997.

[DS97] A. Delshams and T.M. Seara. Splitting of separatrices in Hamiltonian systems with one and a half degrees of freedom. Math. Phys. Electron. J., 3(4):1-40, 1997.

[Eli94] L.H. Eliasson. Biasymptotic solutions of perturbed integrable Hamiltonian systems. Bol. Soc. Bras. Mat, 25(1):57-76, 1994.

[Gal94] G. Gallavotti. Twistless KAM tori, quasi flat homoclinic intersections, and other cancellations in the perturbation series of certain completely integrable Hamiltonian systems. A review. Rev. Math. Phys., 6(3):343-411, 1994. 
[GGM99] G. Gallavotti, G. Gentile, and V. Mastropietro. Separatrix splitting for systems with three time scales. Comm. Math. Phys., 202(1):197-236, 1999.

[Gra74] S.M. Graff. On the conservation of hyperbolic invariant tori for Hamiltonian systems. J. Differential Equations, 15:1-69, 1974.

[HM82] P. Holmes and J.E. Marsden. Melnikov's method and Arnold diffusion for perturbations of integrable Hamiltonian systems. J. Math. Phys., 23:669-675, 1982.

[Kol78] A.N. Kolmogorov. The general theory of dynamical systems and classical mechanics. In Foundations of mechanics [AM78], pages 741-757 (appendix). The original version appeared in 1954 .

[LM88] P. Lochak and C. Meunier. Multiphase averaging for classical systems: with applications to adiabatic theorems, volume 72 of Appl. Math. Sci. Springer-Verlag, New York, 1988.

[LMS99] P. Lochak, J.-P. Marco, and D. Sauzin. On the splitting of invariant manifolds in multidimensional near-integrable Hamiltonian systems. Preprint, 1999.

[Loc92] P. Lochak. Canonical perturbation theory via simultaneous approximation. Russian Math. Surveys, 47(6):57-133, 1992.

[Mos56] J. Moser. The analytic invariants of an area-preserving mapping near a hyperbolic fixed point. Comm. Pure Appl. Math., 9:673-692, 1956.

[Nie99] L. Niederman. Dynamics around simple resonant tori in nearly integrable Hamiltonian systems (to appear in J. Differential Equations). Preprint, 1999.

[Pös82] J. Pöschel. Integrability of Hamiltonian systems on Cantor sets. Comm. Pure Appl. Math., 35:653$696,1982$.

[PT99] A. Pronin and D. Treschev. Continuous averaging in multi-frequency slow-fast systems. Preprint, 1999.

[Rob88] C. Robinson. Horseshoes for autonomous Hamiltonian systems using the Melnikov integral. Ergodic Theory Dynam. Systems, 8:395-409, 1988.

[Rüs75] H. Rüssmann. On optimal estimates for the solutions of linear partial differential equations of first order with constant coefficients on the torus. In J. Moser, editor, Dynamical systems, theory and applications, volume 38 of Lect. Notes in Phys., pages 598-624. Springer-Verlag, New York, 1975.

[RW97] M. Rudnev and S. Wiggins. KAM theory near multiplicity one resonant surfaces in perturbations of a-priori stable Hamiltonian systems. J. Nonlinear Sci., 7:177-209, 1997.

[RW98] M. Rudnev and S. Wiggins. Existence of exponentially small separatrix splittings and homoclinic connections between whiskered tori in weakly hyperbolic near-integrable Hamiltonian systems. Phys. D, 114:3-80, 1998.

[RW99] M. Rudnev and S. Wiggins. On a homoclinic splitting problem. Preprint, 1999.

[Sau99] D. Sauzin. A new method for measuring the splitting of invariant manifolds. Preprint, 1999.

[Sim94] C. Simó. Averaging under fast quasiperiodic forcing. In J. Seimenis, editor, Hamiltonian Mechanics: Integrability and Chaotic Behavior, volume 331 of NATO ASI Ser. B: Phys., pages 13-34. Held in Toruń, Poland, 28 June-2 July 1993. Plenum, New York, 1994.

[Tre91] D. Treschev. The mechanism of destruction of resonance tori of Hamiltonian systems. Math. USSR Sb., 68(1):181-203, 1991.

[Tre94] D. Treschev. Hyperbolic tori and asymptotic surfaces in Hamiltonian systems. Russian J. Math. Phys., 2(1):93-110, 1994.

[Val98] E. Valdinoci. Whiskered transition tori for a priori stable and unstable Hamiltonian systems. Preprint 98-560, mp_arc@math .utexas.edu, 1998.

[Wig88] S. Wiggins. Global bifurcations and chaos: analytical methods, volume 73 of Appl. Math. Sci. SpringerVerlag, New York, 1988.

[Zeh76] E. Zehnder. Generalized implicit function theorems with applications to some small divisor problems/II. Comm. Pure Appl. Math., 29:49-111, 1976.

Internet access: The authors' quoted preprints are available at http://www-ma1.upc.es in the Preprints pages, or at ftp://ftp-ma1.upc.es in the pub/preprints directory. 